\title{
Soft gluon emission at two loops in full color
}

\author{
Lance J. Dixon, ${ }^{a}$ Enrico Herrmann, ${ }^{a}$ Kai Yan $^{b}$ and Hua Xing Zhu ${ }^{c}$ \\ ${ }^{a}$ SLAC National Accelerator Laboratory, Stanford University, \\ Stanford, CA 94039, U.S.A. \\ ${ }^{b}$ Max-Planck-Institut für Physik, Werner-Heisenberg-Institut, \\ Föhringer Ring 6, D-80805 München, Germany \\ ${ }^{c}$ Zhejiang Institute of Modern Physics, Department of Physics, Zhejiang University, \\ 38 Zheda Road, Hangzhou, 310027, China \\ E-mail: lance@slac.stanford.edu, eh10@stanford.edu, kyan@mpp.mpg.de, \\ zhuhx@zju.edu.cn
}

ABSTRACT: The soft emission factor is a central ingredient in the factorization of generic $n$-particle gauge theory amplitudes with one soft gluon in the external state. We present the complete two-loop soft factor, capturing the leading power behavior in the soft-gluon momentum. At two loops, the color structure and the kinematic dependence of the soft factor become nontrivial as the soft gluon can couple to three hard partons for the first time (tripole terms). The nontrivial kinematic dependence of the tripole terms is of uniform, maximal transcendental weight, and can be expressed (in a "Euclidean" region) in terms of single-valued harmonic polylogarithms. Our results are consistent with the behavior of the recently computed symbol of the two-loop five-particle amplitude in $\mathcal{N}=4$ super-YangMills theory. In the limit where the outgoing soft gluon is also collinear with an incoming hard parton, potentially dangerous factorization-violating terms can arise.

KeYwords: Perturbative QCD, Scattering Amplitudes

ArXiv EPrint: 1912.09370 


\section{Contents}

1 Introduction 1

2 Soft factorization $\quad 3$

2.1 Soft factorization at one loop 5

3 Soft factorization at two loops $\quad 6$

3.1 The tripole contribution 8

3.2 Alternate representation of tripole contribution 11

$\begin{array}{ll}3.3 \text { Analytic continuation } & 14\end{array}$

4 Applications $\quad 18$

$\begin{array}{lll}\text { 4.1 Soft limits of scattering amplitudes } & 18\end{array}$

$\begin{array}{lll}4.2 & \text { Soft-collinear limit } & 19\end{array}$

$\begin{array}{llr}5 & \text { Conclusions } & 27\end{array}$

\section{Introduction}

Scattering amplitudes are some of the most intensively studied quantities in gauge theory. On the one hand, they are essential building blocks for precision QCD predictions at high energy colliders, such as the Large Hadron Collider. On the other hand, their analytic structure often reveals hidden properties of gauge theory which are otherwise hard to probe [1-3]. Due to their fundamental importance, there have been significant efforts to understand and exploit the analytic properties of scattering amplitudes.

Multi-loop scattering amplitudes are complicated functions of the momenta and helicities of the external particles. In certain kinematical regimes, gauge theory amplitudes become simpler, by factorizing into products of full lower-point amplitudes [4], or into lower-point amplitudes multiplied by a universal emission factor. Examples of the latter type include collinear factorization [4-6], where two or multiple partons become collinear, or soft factorization, where one or more partons become soft [7-9]. Factorization is of great theoretical interest for several reasons:

a) The computation of perturbative cross sections in gauge theory at higher order in the coupling constant often involves phase space integrals over emission factors arising in the factorization limit [10-13].

b) Certain observables exhibit large logarithms at fixed order, invalidating a simple perturbative calculation. Accurately describing these observables requires resumming the large logarithms, which relies on factorization [14-16]. 
c) In some cases, complete scattering amplitudes can be "bootstrapped" from their asymptotic factorization behavior [17-20].

In this work, we focus on the leading power behavior of gauge theory amplitudes when a single soft gluon is emitted. At tree level, this corresponds to the simple and wellknown eikonal approximation [21]. At one loop, due to infrared divergences, the soft factor receives perturbative corrections [9, 22-24], which can couple at most two hard partons at this order. At two loops, the soft factor has been known for some time for collision processes with two hard colored external states [25, 26]; the same function also describes soft emission in the (planar) limit of a large number of colors $N_{c}$ for gauge group $\mathrm{SU}\left(N_{c}\right)$. However, starting at two loops, it is possible to couple up to three hard particles in the single soft emission process, in a non-planar fashion that contributes at subleading order in $N_{c}$. One of the main novel results in this paper is the computation of this additional "tripole" contribution to the soft factor.

Because the tripole contribution contains only soft gluons, it is the same in any gauge theory at the lowest order of its appearance, namely two loops in perturbation theory. In particular, it is the same in QCD as in $\mathcal{N}=4$ super-Yang-Mills theory (SYM). Therefore the "uniform transcendentality" properties of $\mathcal{N}=4$ SYM amplitudes [27-30] imply similar properties for the universal tripole contributions computed here. (The non-tripole contributions depend on the matter content of the theory and do not have uniform transcendentality at two loops $[25,26]$.) Also, we can employ our results to verify the soft-gluon limit of the full-color two-loop five-particle amplitude in $\mathcal{N}=4 \mathrm{SYM}$, which was computed recently at the level of the symbol [31, 32].

By considering soft-gluon emission in a direction collinear to one of the external hard particles, we can access the soft limit of the collinear splitting amplitudes. In particular, we obtain results in the spacelike splitting regime which are so far, apart from the singular terms, unknown. Given the one- and two-loop soft factor in the spacelike collinear limit, we observe the breaking of strict collinear factorization at next-to-next-to-leading order parton splitting. Such factorization breaking has been observed previously for splitting amplitudes at the level of infrared-divergent terms, which were argued to cancel in color-summed, squared QCD matrix elements [33,34] at this order (although perhaps not at the next order [34-36]). Here we extend these results to the infrared-finite terms in dimensionally-regularized amplitudes and squared splitting probabilities. We find that potential factorization-violating terms survive at the level of the cross section.

The remainder of this work is structured as follows: in section 2 we briefly summarize the relevant features of soft-gluon factorization, and define our conventions while reviewing the known tree-level and one-loop results. Section 3 constitutes the central part of this paper. First, we define the decomposition of the two-loop soft factor into dipole terms (known in the literature) and tripole terms. After summarizing the relevant tripole kinematics, the new pieces are given in one representation in section 3.1, in a "Euclidean" kinematic configuration. We provide an alternate representation for the dipole terms in section 3.2, eqs. (3.15) and (3.16), still in the Euclidean region. In section 3.3, we define the analytic continuation to all other kinematic configurations. This continuation will play 
a role in our investigation of strict collinear factorization violation at cross-section level, as we take the soft factor into a (spacelike) collinear limit in section 4.2. We also mention the relevance of the soft factor for non-planar two-loop five-particle amplitudes in section 4.1, and finally we conclude in section 5 . We provide a supplementary material containing computer-readable versions of some of the lengthier expressions in this paper.

\section{Soft factorization}

Consider a scattering process with $n+1$ all outgoing ${ }^{1}$ colored partons and any number of color-neutral particles,

$$
0 \rightarrow p_{1}+p_{2}+\cdots+p_{n}+q+\text { color neutral particles },
$$

where $q$ denotes the momentum of the soft gluon. In the limit where $q$ becomes soft, i.e. $q \cdot p_{i}$ is parametrically smaller than $p_{i} \cdot p_{j}$ for all $1 \leq i, j \leq n$, the (bare, unrenormalized) amplitude for this process factorizes into the product of a singular soft factor and an amplitude omitting the soft gluon: ${ }^{2}$

$$
\left|\mathcal{M}_{n+1}\left(\left\{p_{m}\right\}, q\right)\right\rangle \simeq \epsilon_{ \pm}^{\mu}(q) \boldsymbol{J}_{\mu}(q)\left|\mathcal{M}_{n}\left(\left\{p_{m}\right\}\right)\right\rangle \equiv \boldsymbol{S}^{ \pm}\left(\left\{\beta_{m}\right\}, q\right)\left|\mathcal{M}_{n}\left(\left\{p_{m}\right\}\right)\right\rangle .
$$

For an $\mathrm{SU}\left(N_{c}\right)$ gauge theory such as QCD, the amplitude can be conveniently represented as a vector in color space [37]. The soft factor, $\boldsymbol{S}^{ \pm}\left(\left\{\beta_{m}\right\}, q\right)$, and likewise the soft current $\boldsymbol{J}_{\mu}(q)$, are color operators passing from an $n$-particle color space to an $(n+1)$-particle color space by adding one soft gluon [24]. In eq. (2.2), the ' $\simeq$ ' sign signals that the equality holds for the leading terms in the soft limit as the gluon's energy vanishes, $q^{0}=|\vec{q}| \rightarrow 0$, up to power corrections in $q^{0}$. Also, ' \pm ' in $\epsilon_{ \pm}(q)$ denotes the polarization (helicity) of the soft gluon. The (leading) soft factorization is universal in the sense that the soft factor is independent of the helicities and flavor of the hard partons in the process; it only depends on the color charge and angular direction of the hard partons, $\beta_{m}^{\mu}=p_{m}^{\mu} / p_{m}^{0}$. (In this work we consider massless partons only, and just the leading powers in $q^{0}$.) As we shall see later, the latter fact is particularly important and is sometimes dubbed "rescaling invariance" [38-43], or "reparameterization invariance" [44].

The $n$-point, all-gluon amplitudes can be expanded perturbatively in the strong coupling constant $g_{s}$,

$$
\left|\mathcal{M}_{n}\left(\left\{p_{m}\right\}\right)\right\rangle=\sum_{L=0}^{\infty} g_{s}^{n-2} \bar{a}^{L}\left|\mathcal{M}_{n}^{(L)}\left(\left\{p_{m}\right\}\right)\right\rangle,
$$

where we have introduced a rescaled coupling,

$$
\bar{a} \equiv \frac{g_{s}^{2}}{(4 \pi)^{2-\epsilon}} e^{-\epsilon \gamma_{E}}=\frac{\alpha_{s}}{4 \pi} \frac{e^{-\epsilon \gamma_{E}}}{(4 \pi)^{-\epsilon}},
$$

where $\alpha_{s}=g_{s}^{2} /(4 \pi)$, and $\gamma_{E}$ is the Euler-Mascheroni constant. Perturbatively expanding the factorization formula (2.2) subsequently defines the perturbative expansion of the soft

\footnotetext{
${ }^{1}$ Processes with incoming colored partons can be obtained from the all-outgoing case by crossing.

${ }^{2}$ We include the definition of the soft current $\boldsymbol{J}_{\mu}(q)$ for convenient comparison with e.g. ref. [26].
} 
factor,

$$
\begin{aligned}
g_{s}^{(n+1)-2} \bar{a}^{L}\left|\mathcal{M}_{n+1}^{(L)}\left(\left\{p_{m}\right\}, q\right)\right\rangle & \simeq \sum_{\ell=0}^{L} g_{s} \bar{a}^{\ell} \boldsymbol{S}^{ \pm,(\ell)}\left(\left\{\beta_{m}\right\}, q\right) \times g_{s}^{n-2} \bar{a}^{L-\ell}\left|\mathcal{M}_{n}^{(L-\ell)}\left(\left\{p_{m}\right\}\right)\right\rangle, \\
\boldsymbol{S}^{ \pm}\left(\left\{\beta_{m}\right\}, q\right) & \equiv \sum_{L=0}^{\infty} g_{s} \bar{a}^{L} \boldsymbol{S}^{ \pm,(L)}\left(\left\{\beta_{m}\right\}, q\right) .
\end{aligned}
$$

It has been shown diagrammatically that eq. (2.2) holds at one loop in ref. [24], and later to all loops in refs. $[45,46]$. In particular, the soft factor can be calculated to all orders as the matrix element of time-ordered Wilson line operators with a single gluon in the external state:

$$
\boldsymbol{S}^{ \pm}\left(\left\{\beta_{m}\right\}, q\right)=\left\langle q\left|\int d^{4} x e^{\mathrm{i} x \cdot q} \mathrm{~T}\left\{\prod_{k=1}^{n} Y_{k}(x)\right\}\right| 0\right\rangle,
$$

where $Y_{k}(x)$ is a semi-infinite Wilson line that acts as a lightlike color source for one of the $n$ hard partons in the external state.

For example, for an outgoing parton with momentum $p_{k}$, its corresponding Wilson line starts from the scattering origin, $x$, and extends to null infinity along the direction of the parton velocity $\beta_{k}$ :

$$
Y_{k}(x)=P \exp \left(\mathrm{i} \frac{g_{s}}{\sqrt{2}} \int_{0}^{\infty} d s \beta_{k} \cdot A^{a}\left(x+s \beta_{k}\right) \boldsymbol{T}_{k}^{a}\right) .
$$

Here, $P$ stands for path ordering, and $\boldsymbol{T}_{k}^{a}$ is the color-charge operator in the color space formalism [37]. For an outgoing quark (incoming anti-quark), $\left(T_{k}^{a}\right)_{\alpha \beta}=\left(t^{a}\right)_{\alpha \beta}$, for an outgoing anti-quark (incoming quark), $\left(T_{k}^{a}\right)_{\alpha \beta}=\left(-t^{a}\right)_{\beta \alpha}$, and for a gluon, $\left(T_{k}^{a}\right)_{\alpha \beta}=f^{\alpha a \beta}$, where $t^{a}$ are the non-standard Gell-Mann matrices normalized according to

$$
\operatorname{tr}\left[t^{a} t^{b}\right]=\delta^{a b} \text {, satisfying the color-algebra }\left[t^{a}, t^{b}\right]=f^{a b c} t^{c} .
$$

Note that the structure constants $f^{a b c}$ used in this paper are $\mathrm{i} \sqrt{2}$ times larger than the conventional ones, and are often denoted by $\tilde{f}^{a b c}$, but here we will drop the ' $\sim$ '. Our normalization of the generators $T_{k}^{a}$ also accounts for the $1 / \sqrt{2}$ in eq. (2.8).

At tree level, the soft current is simply the well-known eikonal factor

$$
\boldsymbol{J}^{a,(0) \mu}(q)=\frac{1}{\sqrt{2}} \sum_{i=1}^{n} \boldsymbol{T}_{i}^{a} \frac{p_{i}^{\mu}}{p_{i} \cdot q}=\frac{1}{\sqrt{2}} \frac{1}{2 n} \sum_{i \neq j=1}^{n}\left(\boldsymbol{T}_{i}^{a}-\boldsymbol{T}_{j}^{a}\right)\left(\frac{p_{i}^{\mu}}{p_{i} \cdot q}-\frac{p_{j}^{\mu}}{p_{j} \cdot q}\right),
$$

which can be shown by using color conservation, $\sum_{i=1}^{n} \boldsymbol{T}_{i}^{a}=0$. The factor of $1 /(2 n)$ removes overcounting.

In terms of spinor helicity variables, the soft factors $\boldsymbol{S}_{a}^{ \pm,(0)}\left(\left\{\beta_{m}\right\}, q\right)=\varepsilon_{ \pm} \cdot \boldsymbol{J}^{a,(0)}(q)$ can be obtained by dotting polarization vectors of the soft gluon,

$$
\varepsilon_{+}^{\nu}(q) \mapsto \varepsilon_{+}^{\alpha \dot{\alpha}}(q)=+\sqrt{2} \frac{\xi^{\alpha} \widetilde{\lambda}_{q}^{\dot{\alpha}}}{\langle q \xi\rangle}, \quad \varepsilon_{-}^{\nu}(q) \mapsto \varepsilon_{-}^{\alpha \dot{\alpha}}(q)=-\sqrt{2} \frac{\lambda_{q}^{\alpha} \widetilde{\xi}^{\dot{\alpha}}}{[q \xi]},
$$




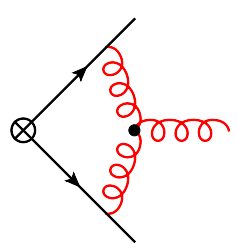

(a)

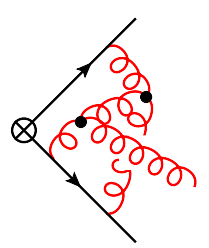

(b)

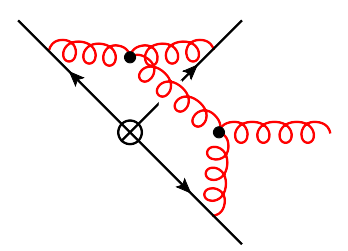

(c)

Figure 1. Representative diagrams for the (a) one-loop, (b) two-loop dipole, and (c) two-loop tripole soft factors.

into the soft current $\boldsymbol{J}^{a,(0) \mu}(q)$. The arbitrary reference spinors $\xi^{\alpha}$ and $\widetilde{\xi}^{\dot{\alpha}}$ entering

$$
\frac{\varepsilon_{+} \cdot p_{i}}{q \cdot p_{i}}-\frac{\varepsilon_{+} \cdot p_{j}}{q \cdot p_{j}}=\frac{\sqrt{2}}{\langle q \xi\rangle}\left[\frac{\langle\xi i\rangle\langle q j\rangle-\langle\xi j\rangle\langle q i\rangle}{\langle q i\rangle\langle q j\rangle}\right]=\sqrt{2} \frac{\langle i j\rangle}{\langle i q\rangle\langle q j\rangle},
$$

drop out in the second equality due to the Schouten identity. The non-standard normalization of the color generators exactly cancels the $\sqrt{2}$ from the polarization vector, yielding the tree-level soft factor in the helicity basis,

$$
\begin{aligned}
& \boldsymbol{S}_{a}^{+,(0)}\left(\left\{\beta_{m}\right\}\right)=+\frac{1}{2 n} \sum_{i \neq j}\left(\boldsymbol{T}_{i}^{a}-\boldsymbol{T}_{j}^{a}\right) \frac{\langle i j\rangle}{\langle i q\rangle\langle q j\rangle}, \\
& \boldsymbol{S}^{-,(0)}\left(\left\{\beta_{m}\right\}\right)=-\frac{1}{2 n} \sum_{i \neq j}\left(\boldsymbol{T}_{i}^{a}-\boldsymbol{T}_{j}^{a}\right) \frac{[i j]}{[i q][q j]} .
\end{aligned}
$$

At tree level, the soft factor is a sum over different gauge-invariant dipole emissions. It is manifestly invariant under rescaling of individual hard parton momenta, $p_{m} \rightarrow e^{t_{m}} p_{m}$, and therefore it only depends on $\beta_{m}$, the angular direction of $p_{m}$. Eq. (2.13) is an important building block for next-to-leading order QCD calculations, and for leading and next-toleading logarithmic resummation. Parity dictates that the emission of a negative-helicity gluon can be obtained from the positive-helicity case by the simple replacement $\langle a b\rangle \rightarrow[b a]$. Therefore, below we will present results only for the soft factor associated with a positivehelicity gluon.

\subsection{Soft factorization at one loop}

At loop level, the soft factor receives corrections. The one-loop soft factor was first obtained in ref. [22] (see also refs. [9, 23]) for color-ordered amplitudes by taking the soft-gluon limit of the one-loop splitting amplitude [5, 9, 22, 23]. It was later re-derived for amplitudes in color space using soft-gluon insertion techniques, i.e. using the eikonal approximation for both external and internal soft gluons [24]. This computation was performed in axial gauge. Since the soft factor is gauge invariant, one can repeat the calculation in Feynman gauge, which is much easier since there is only one non-vanishing diagram involved [25], shown in figure 1a. The result is again a sum over gauge-invariant dipole emissions (see also ref. [26]):

$$
\boldsymbol{S}_{a}^{+,(1)}=\frac{1}{2} C_{1}(\epsilon) \sum_{i \neq j} f_{a a_{i} a_{j}} \boldsymbol{T}_{i}^{a_{i}} \boldsymbol{T}_{j}^{a_{j}} V_{i j}^{q} \frac{\langle i j\rangle}{\langle i q\rangle\langle q j\rangle},
$$


where

$$
\begin{aligned}
C_{1}(\epsilon) & =-\frac{e^{\epsilon \gamma_{E}}}{\epsilon^{2}} \frac{\Gamma^{3}(1-\epsilon) \Gamma^{2}(1+\epsilon)}{\Gamma(1-2 \epsilon)}=-\frac{1}{\epsilon^{2}}-\frac{\zeta_{2}}{2}+\epsilon \frac{7}{3} \zeta_{3}+\epsilon^{2} \frac{39}{16} \zeta_{4}+\mathcal{O}\left(\epsilon^{3}\right), \\
V_{i j}^{q} & =\left[\frac{\mu^{2}\left(-s_{i j}\right)}{\left(-s_{i q}\right)\left(-s_{q j}\right)}\right]^{\epsilon} .
\end{aligned}
$$

We define the conventional Mandelstam variables

$$
s_{a b}=\langle a b\rangle[b a]=-2\left|p_{a} \cdot p_{b}\right| \exp \left(-\mathrm{i} \pi \lambda_{a b}\right),
$$

with $\lambda_{a b}=1$ if $a$ and $b$ are both incoming or both outgoing, and 0 otherwise. Again, the one-loop soft factor is explicitly invariant under rescaling of the hard parton momenta. The analytic dependence on the parton momenta is fully captured by $V_{i j}^{q}$, which is itself uniquely fixed by dimensional analysis and rescaling symmetry, given that only two hard partons can be involved in a non-factorizable one-loop diagram. The analytic continuation of eq. (2.16) to any kinematic region is given by eq. (2.17), which is equivalent to letting

$$
\log \left(-s_{a b}\right) \rightarrow \log \left|s_{a b}\right|-i \pi \Theta\left(s_{a b}\right)
$$

where $\Theta(x)$ is the Heaviside theta function, after expanding eq. (2.16) in $\epsilon$.

We emphasize that eq. (2.14) is the one-loop soft emission factor for bare, unrenormalized amplitudes; one can also define an emission factor for renormalized amplitudes but it will differ by a term proportional to the tree emission factor multiplied by the beta function coefficient $\beta_{0}$. In the above unrenormalized case, the one-loop soft factor is purely non-Abelian, which is in agreement with the expectation [21] that the soft photon limit in an Abelian gauge theory with no massless charged fermions should be exact. If there are massless charged fermions, there is a correction proportional to $\beta_{0}$ to the soft emission factor for renormalized amplitudes, but there is no correction at one loop to unrenormalized Abelian amplitudes.

\section{Soft factorization at two loops}

At two loops, the soft factor becomes substantially more involved. In the large $N_{c}$ limit, also allowing for a large number of fermion flavors $N_{f}$, the soft factor again factorizes into a sum of gauge-invariant dipole emissions. The soft factor in this limit was first obtained by taking the soft limit of the two-loop planar splitting amplitude [47]. At higher orders in $\epsilon$, it has also been obtained by evaluating the soft limit of the integrals entering the two-loop amplitude for $e^{+} e^{-} \rightarrow q \bar{q} g$ [26], and by direct calculation using eikonal techniques [25].

One of the main results of this work is the computation of the two-loop non-planar contribution to the soft factor. In contrast to tree level and one loop, the soft factor contains contributions from tripole emission as well as dipole emission:

$$
\boldsymbol{S}_{a}^{+,(2)}=\frac{1}{2} \sum_{i \neq j} \boldsymbol{S}_{a, i j}^{+,(2)}-\frac{1}{4} \sum_{i \neq k \neq j} \boldsymbol{S}_{a, i k j}^{+,(2)} .
$$


Tripole emission is characterized by three separate hard legs, $i, j, k$. Representative Feynman diagrams contributing to dipole and tripole emission are depicted in figure $1 \mathrm{~b}$ and $1 \mathrm{c}$, respectively. The calculation is similar to the one in ref. [25]. First, we generate all Feynman diagrams for the Wilson line matrix elements with a soft gluon using QGRAF [48]. After performing color and Dirac algebra manipulations, the diagrams are passed to LiteRed [49] for Integration-By-Parts [50] (IBP) reduction. Since the dipole integrals are already known [25], we focus on the tripole topology. Here, the IBP reduction leads to 8 master integrals in total, which (owing to the nontrivial kinematic dependence) we solve using the method of differential equations [51-53].

The result for the dipole emission is given by

$$
\boldsymbol{S}_{a, i j}^{+,(2)}=C_{2}(\epsilon) f_{a a_{i} a_{j}} \boldsymbol{T}_{i}^{a_{i}} \boldsymbol{T}_{j}^{a_{j}}\left(V_{i j}^{q}\right)^{2} \frac{\langle i j\rangle}{\langle i q\rangle\langle q j\rangle},
$$

where $C_{2}(\epsilon)$ is a constant which can be expanded in $\epsilon$ :

$$
\begin{aligned}
C_{2}(\epsilon)= & C_{A}\left[\frac{1}{2 \epsilon^{4}}-\frac{11}{12 \epsilon^{3}}+\frac{1}{\epsilon^{2}}\left(\zeta_{2}-\frac{16}{9}-\frac{\delta_{R}}{12}\right)-\frac{1}{\epsilon}\left(\frac{11 \zeta_{3}}{6}+\frac{11 \zeta_{2}}{12}+\frac{181}{54}+\frac{2 \delta_{R}}{9}\right)\right. \\
& \left.+\frac{7 \zeta_{4}}{8}+\frac{341 \zeta_{3}}{18}-\frac{16 \zeta_{2}}{9}-\frac{\delta_{R} \zeta_{2}}{12}-\frac{1037}{162}-\frac{35 \delta_{R}}{54}\right] \\
+ & T_{R} N_{f}\left[\frac{1}{3 \epsilon^{3}}+\frac{5}{9 \epsilon^{2}}+\frac{1}{\epsilon}\left(\frac{\zeta_{2}}{3}+\frac{19}{27}\right)-\frac{62 \zeta_{3}}{9}+\frac{5 \zeta_{2}}{9}+\frac{65}{81}\right] \\
+ & C_{A} N_{s}\left[\frac{1}{24 \epsilon^{3}}+\frac{1}{9 \epsilon^{2}}+\frac{1}{\epsilon}\left(\frac{\zeta_{2}}{24}+\frac{35}{108}\right)-\frac{31 \zeta_{3}}{36}+\frac{\zeta_{2}}{9}+\frac{259}{324}\right]+\mathcal{O}(\epsilon) .
\end{aligned}
$$

This result is presented for one adjoint vector, $N_{f}$ flavors of fundamental fermions, and $N_{s}$ flavors of adjoint real scalars. The parameter $\delta_{R}$ selects the regularization scheme, where $\delta_{R}=1$ corresponds to the 't Hooft-Veltman scheme [54], and $\delta_{R}=0$ corresponds to the Four-Dimensional-Helicity scheme $[55,56]$ (which preserves supersymmetry here). In $\mathrm{SU}\left(N_{c}\right)$ gauge theory, $C_{A}=N_{c}$, and $T_{R}=1 / 2$. If we set $\delta_{R}=1$ and $N_{s}=0$, and take into account different normalization conventions, then the result (3.3) agrees with ref. [47], and also with the terms through $\mathcal{O}\left(\epsilon^{0}\right)$ in refs. $[25,26]$. The latter references give results valid to $\mathcal{O}\left(\epsilon^{2}\right)$ and to all orders in $\epsilon$, respectively, for the dipole terms in QCD.

The corresponding result in $\mathcal{N}=4 \mathrm{SYM}$ can be obtained by setting $T_{R} \rightarrow C_{A} / 2$, $N_{f}=4$, and $N_{s}=6$. For $\delta_{R}=0$, the $\mathcal{N}=4 \mathrm{SYM}$ result has uniform transcendentality,

$$
C_{2}^{\mathcal{N}}=4(\epsilon)=C_{A}\left[\frac{1}{2 \epsilon^{4}}+\frac{\zeta_{2}}{\epsilon^{2}}-\frac{11 \zeta_{3}}{6 \epsilon}+\frac{7 \zeta_{4}}{8}\right],
$$

and it agrees with the soft $(z \rightarrow 0)$ limit of the two-loop splitting amplitude in planar $\mathcal{N}=4$ SYM [57].

We note that the factor $C_{2}(\epsilon)$ in eq. (3.3) contains no explicit subleading-in- $N_{c}$ terms. However, there are still non-planar corrections to the dipole contribution. In fact, both of the non-planar diagrams in figures $1 \mathrm{~b}$ and $1 \mathrm{c}$ give rise to dipole terms. In particular, the 
dipole contribution from figure 1c comes from summing over color indices and applying the color Jacobi identity. This statement is consistent with eq. (3.2), in which the color operator gives both leading- and subleading-color contributions when acting on an amplitude with more than two colored hard partons.

\subsection{The tripole contribution}

Before presenting the result for the tripole contribution to the soft emission factor $\boldsymbol{S}_{a, i k j}^{+,(2)}$, it is useful to comment on the relevant kinematics. For a given tripole of hard lines $(i, j, k)$, the soft factor can only depend on $V_{i j}^{q}$ and rescaling-invariant cross ratios constructed from $p_{i}, p_{j}, p_{k}$ and $q$. From the four momenta involved in the problem, one can form two independent cross ratios:

$$
u_{k}^{i j} \equiv \frac{s_{i k} s_{j q}}{s_{i j} s_{k q}}, \quad v_{k}^{i j} \equiv \frac{s_{j k} s_{i q}}{s_{i j} s_{k q}} .
$$

It turns out that the two-loop tripole emission contribution is a complicated function of $u_{k}^{i j}$ and $v_{k}^{i j}$. In particular, it depends on the following Källén function:

$$
\Delta_{k}^{i j}=\sqrt{1-2 u_{k}^{i j}-2 v_{k}^{i j}+\left(u_{k}^{i j}-v_{k}^{i j}\right)^{2}}
$$

which gives rise to complications with the analytic continuation. To rationalize the kinematics, we use the well-known parameterization of the cross ratios:

$$
u_{k}^{i j}=\left(1-z_{k}^{i j}\right)\left(1-\bar{z}_{k}^{i j}\right), \quad v_{k}^{i j}=z_{k}^{i j} \bar{z}_{k}^{i j},
$$

where $z_{k}^{i j}$ and $\bar{z}_{k}^{i j}$ are cross ratios of spinor products,

$$
z_{k}^{i j}=\frac{\langle k j\rangle\langle i q\rangle}{\langle i j\rangle\langle k q\rangle}, \quad \bar{z}_{k}^{i j}=\frac{[k j][i q]}{[i j][k q]} .
$$

Focusing on the holomorphic variables, it is useful to keep in mind that $z_{k}^{i j}$ satisfies the Schouten identity,

$$
1-z_{k}^{i j}=1-\frac{\langle k j\rangle\langle i q\rangle}{\langle i j\rangle\langle k q\rangle}=\frac{\langle k i\rangle\langle j q\rangle}{\langle j i\rangle\langle k q\rangle}=z_{k}^{j i} .
$$

The change of variables in eq. (3.7) sets

$$
\Delta_{k}^{i j}=z_{k}^{i j}-\bar{z}_{k}^{i j}=\frac{[i j]\langle j k\rangle[k q]\langle q i\rangle-\langle i j\rangle[j k]\langle k q\rangle[q i]}{\langle i j\rangle[j i]\langle k q\rangle[q k]}=\frac{4 \mathrm{i} \varepsilon\left(p_{i}, p_{j}, p_{k}, q\right)}{s_{i j} s_{k q}},
$$

where $\varepsilon\left(p_{i}, p_{j}, p_{k}, q\right)$ is the contracted Levi-Civita tensor. Repeated use of the Schouten identity leads to four more relations,

$$
z_{i}^{k j}=\frac{1}{z_{k}^{i j}}, \quad z_{i}^{j k}=\frac{1-z_{k}^{i j}}{-z_{k}^{i j}}, \quad z_{j}^{i k}=\frac{-z_{k}^{i j}}{1-z_{k}^{i j}}, \quad z_{j}^{k i}=\frac{1}{1-z_{k}^{i j}},
$$

which will be useful in summing the tripole soft factor.

The change of variables (3.7) can be motivated by a stereographic projection, under which a lightlike momentum is mapped to a point on the complex plane (see 


\begin{tabular}{|c|ll|}
\hline$A_{0}$ : all outgoing & $u_{k}^{i j}=\left|u_{k}^{i j}\right|$ & $v_{k}^{i j}=\left|v_{k}^{i j}\right|$ \\
\hline$A_{1}: j, k$ incoming, $i, q$ outgoing & $u_{k}^{i j} \rightarrow\left|u_{k}^{i j}\right|$ & $v_{k}^{i j} \rightarrow\left|v_{k}^{i j}\right| e^{-2 \mathrm{i} \pi}$ \\
\hline$A_{2}: i, k$ incoming, $j, q$ outgoing & $u_{k}^{i j} \rightarrow\left|u_{k}^{i j}\right| e^{-2 \mathrm{i} \pi}$ & $v_{k}^{i j} \rightarrow\left|v_{k}^{i j}\right|$ \\
\hline$A_{3}: i, j$ incoming, $k, q$ outgoing & $u_{k}^{i j} \rightarrow\left|u_{k}^{i j}\right| e^{2 \mathrm{i} \pi}$ & $v_{k}^{i j} \rightarrow\left|v_{k}^{i j}\right| e^{2 \mathrm{i} \pi}$ \\
\hline$A_{4}: i$ incoming, $j, k, q$ outgoing & $u_{k}^{i j} \rightarrow\left|u_{k}^{i j}\right|$ & $v_{k}^{i j} \rightarrow\left|v_{k}^{i j}\right|$ \\
\hline$A_{5}: j$ incoming, $i, k, q$ outgoing & $u_{k}^{i j} \rightarrow\left|u_{k}^{i j}\right|$ & $v_{k}^{i j} \rightarrow\left|v_{k}^{i j}\right|$ \\
\hline$A_{6}: k$ incoming, $i, j, q$ outgoing & $u_{k}^{i j} \rightarrow\left|u_{k}^{i j}\right|$ & $v_{k}^{i j} \rightarrow\left|v_{k}^{i j}\right|$ \\
\hline$A_{7}: i, j, k$ incoming, $q$ outgoing & $u_{k}^{i j} \rightarrow\left|u_{k}^{i j}\right|$ & $v_{k}^{i j} \rightarrow\left|v_{k}^{i j}\right|$ \\
\hline
\end{tabular}

Table 1. Rules for analytically continuing $u_{k}^{i j}$ and $v_{k}^{i j}$ from region $A_{0}$, also dubbed the "Euclidean" region, to other physical regions. These rules can be obtained from eqs. (2.17) and (3.5). The rules are used below to continue the tripole emission terms to generic physical regions.

e.g. refs. $[58,59])$, and spinor products map to differences of complex coordinates. The $\mathrm{SL}(2, \mathbb{C})$ invariance of the cross ratios can then be used to map the points corresponding to $p_{i}, p_{j}, p_{k}$ to $0,1, \infty$ on the complex plane, and the point corresponding to $q$ maps to $z_{k}^{i j}$. In this chart, the limits $z_{k}^{i j} \rightarrow 0,1, \infty$ correspond to the collinear limits in which $q$ becomes parallel to $p_{i}, p_{j}, p_{k}$, respectively. On the real axis $\left(\operatorname{Im}\left(z_{k}^{i j}\right)=0\right.$, eq. (3.10)) the volume element of $p_{i}, p_{j}, p_{k}$ and $q$ vanishes $\left(\varepsilon\left(p_{i}, p_{j}, p_{k}, q\right)=0\right)$ so that the momenta lie in a lower-dimensional subspace. Parity exchanges $\langle a b\rangle \leftrightarrow[b a]$, so it maps $\Delta_{k}^{i j} \leftrightarrow-\Delta_{k}^{i j}$ and is implemented as complex conjugation, $z_{k}^{i j} \leftrightarrow \bar{z}_{k}^{i j}$.

We shall first present the result for the tripole emission soft factor in a "Euclidean" region $^{3}$ in which all momenta participating in the tripole, including the soft momentum, are either all incoming or all outgoing, so that $u_{k}^{i j}>0$ and $v_{k}^{i j}>0$. This region is denoted by $A_{0}$ in table 1 . Then we shall explain how to obtain the results in various physical regions by analytic continuation, as summarized in table 1 , where the phase factors follow from eqs. (2.17) and (3.5). In principle there are $2^{4}=16$ physical regions for each tripole, since the three hard momenta $p_{i}, p_{j}, p_{k}$ and the soft momentum $q$ can be incoming or outgoing. However, if all incoming and outgoing momenta are swapped with each other, the Mandelstam variables do not change. Hence it suffices to give the eight configurations with $q$ outgoing, as shown in table 1 . Also, the cases $A_{4}$ to $A_{7}$, with an odd number of incoming momenta and an odd number of outgoing ones, always have one timelike and one spacelike invariant each in the numerator and in the denominator of each cross ratio, so the phases cancel and no continuation from $A_{0}$ is required. Only cases $A_{1}, A_{2}$ and $A_{3}$ require a nontrivial continuation.

The tripole emission contribution can be decomposed into rational prefactors multiplying transcendental functions. The rational prefactors are essentially the tree-level emission factors (2.12). In principle there are three such factors, for the three pairs of legs entering the tripole, $i j, j k$ and $i k$, but the Schouten identity allows the $i j$ factor to be eliminated,

\footnotetext{
${ }^{3}$ Depending on the momenta not involved in the tripole emission, this region could still correspond to a physical scattering region.
} 
leaving us with

$$
\boldsymbol{S}_{a, i k j}^{+,(2)}=\left(V_{i j}^{q}\right)^{2} f^{a a_{k} b} f^{b a_{i} a_{j}} \boldsymbol{T}_{i}^{a_{i}} \boldsymbol{T}_{j}^{a_{j}} \boldsymbol{T}_{k}^{a_{k}}\left[\frac{\langle i k\rangle}{\langle i q\rangle\langle q k\rangle} F\left(z_{k}^{i j}, \epsilon\right)-\frac{\langle j k\rangle}{\langle j q\rangle\langle q k\rangle} F\left(z_{k}^{j i}, \epsilon\right)\right] .
$$

The transcendental function $F\left(z_{k}^{i j}, \epsilon\right) \equiv F(z, \epsilon)$ is a function of $z$ and $\bar{z}$ on the complex plane. Note that Bose symmetry under exchange of legs $i$ and $j$ is manifest within a single tripole (3.12), whereas symmetry under other exchanges, such as $j$ and $k$, requires summing over different tripoles. In the $A_{0}$ region, the soft factor cannot develop branch cuts in the kinematic variables, unless one approaches the boundaries, corresponding to Mandelstam variables vanishing, or equivalently, $u_{k}^{i j}$ and $v_{k}^{i j}$ going to 0 or infinity. These branch cuts are not in $z$, but only in $|z|^{2}$ or $|1-z|^{2}$. Such functions are called real analytic functions, or single-valued functions of $z$ (with $\bar{z}=z^{*}$ ). If they are polylogarithmic, then the first entries of their symbol [60] can only be $z \bar{z}$ or $(1-z)(1-\bar{z})[61]$.

It turns out that through $\mathcal{O}\left(\epsilon^{0}\right)$, the tripole emission contribution can be fully described by a special class of such real analytic functions, called single-valued harmonic polylogarithms (SVHPLs) [62, 63]. Interestingly, such functions also appear in the context of the soft-gluon anomalous dimension at three loops [43, 59]. In that case, SVHPLs with transcendental weight up to 5 can appear, while here we encounter at most weight 4 . The SVHPLs related to our problem are denoted by $\mathcal{L}_{\vec{w}}(z, \bar{z})$, where $\vec{w}$ is a binary string of 0's and 1's of length $|w|$ equal to the weight. The $\bar{z}$ dependence will often be implicit below. They are defined by the differential equations

$$
\frac{\partial}{\partial z} \mathcal{L}_{0, \vec{w}}(z, \bar{z})=\frac{\mathcal{L}_{\vec{w}}(z, \bar{z})}{z}, \quad \frac{\partial}{\partial z} \mathcal{L}_{1, \vec{w}}(z, \bar{z})=\frac{\mathcal{L}_{\vec{w}}(z, \bar{z})}{1-z}
$$

subject to the constraints of single-valuedness and

$$
\mathcal{L}_{0^{n}}(z, \bar{z})=\frac{1}{n !} \log ^{n}|z|^{2}, \quad n \geq 0, \quad \mathcal{L}_{1}(z, \bar{z})=-\log |1-z|^{2}, \quad \lim _{z \rightarrow 0} \mathcal{L}_{\vec{w} \neq 0^{n}}(z, \bar{z})=0
$$

An explicit construction of SVHPLs in terms of the more familiar harmonic polylogarithms (HPLs) [64] is given in ref. [63]. SVHPLs have also been implemented in the Mathematica package PolyLogTools [65] which allows easy manipulation of expressions containing SVHPLs.

As mentioned in the introduction, the tripole contribution to the two-loop soft factor contains only soft gluons and is therefore the same in any gauge theory, including $\mathcal{N}=4$ SYM. Thanks to the uniform transcendentality property of $\mathcal{N}=4 \mathrm{SYM}$, we expect $F(z, \epsilon)$ to be a function of uniform weight 4 (counting $\epsilon$ with transcendental weight -1 ), and 
indeed it is: ${ }^{4}$

$$
\begin{aligned}
F(z, \epsilon)= & \frac{1}{\epsilon^{2}} \mathcal{L}_{0} \mathcal{L}_{1}+\frac{1}{3 \epsilon}\left(\mathcal{L}_{0}^{2} \mathcal{L}_{1}-2 \mathcal{L}_{0} \mathcal{L}_{1}^{2}\right) \\
& -\mathcal{L}_{1}\left(\frac{2}{9} \mathcal{L}_{0}^{3}+\frac{1}{3} \mathcal{L}_{0}^{2} \mathcal{L}_{1}+\frac{13}{18} \mathcal{L}_{0} \mathcal{L}_{1}^{2}+\frac{7}{12} \mathcal{L}_{1}^{3}\right)+2 \mathcal{L}_{1,0,1,0} \\
& +\frac{4}{3}\left[2\left(\mathcal{L}_{0,0,0,1}+\mathcal{L}_{0,0,1,0}\right)+\mathcal{L}_{0,0,1,1}-\mathcal{L}_{0,1,1,1}-\mathcal{L}_{1,0,1,1}-\mathcal{L}_{1,1,0,0}\right] \\
& +2 \zeta_{2}\left(2 \mathcal{L}_{0,1}-\mathcal{L}_{0} \mathcal{L}_{1}\right)+\frac{40}{3} \zeta_{3} \mathcal{L}_{1}+\mathcal{O}(\epsilon)
\end{aligned}
$$

where $\mathcal{L}_{\vec{w}}=\mathcal{L}_{\vec{w}}(z, \bar{z})$. Explicit expressions for $F(z, \epsilon)$ in terms of harmonic polylogarithms in $z$ and $\bar{z}$, and in terms of $G$ functions, as well as its symbol, are provided in the supplementary material.

\subsection{Alternate representation of tripole contribution}

In this subsection we provide another representation of the contributions of a single tripole $\{i, j, k\}$, which is more convenient for many purposes, including describing the analytic continuation into regions $A_{1}, A_{2}$, and $A_{3}$. It turns out that the single term $\boldsymbol{S}_{a, i k j}^{+,(2)}$ given in eq. (3.12) becomes ambiguous in the kinematic regions $A_{1}, A_{2}$, and $A_{3}$. The ambiguities cancel, as they must, after summing over all six permutations of $i, j, k$ corresponding to a given tripole.

Thus we wish to rewrite the second term of eq. (3.1) as a sum over distinct tripoles, labelled by the unordered set $\{i, j, k\}$ :

$$
-\frac{1}{4} \sum_{i \neq k \neq j} \boldsymbol{S}_{a, i k j}^{+,(2)}=-\frac{1}{4} \sum_{\substack{\text { tripoles } \\\{i, j, k\}}} \boldsymbol{S}_{a,\{i, j, k\}}^{+,(2)},
$$

where

$$
\begin{aligned}
& \boldsymbol{S}_{a,\{i, j, k\}}^{+,(2)}=2\left(\boldsymbol{S}_{a, i k j}^{+,(2)}+\boldsymbol{S}_{a, k j i}^{+,(2)}+\boldsymbol{S}_{a, j i k}^{+,(2)}\right) \\
&=2 \boldsymbol{T}_{i}^{a_{i}} \boldsymbol{T}_{j}^{a_{j}} \boldsymbol{T}_{k}^{a_{k}}\left\{\frac{\langle i k\rangle}{\langle i q\rangle\langle q k\rangle}\left(V_{i k}^{q}\right)^{2}\left[f^{a a_{j} b} f^{b a_{i} a_{k}} D_{1}(z, \bar{z})+f^{a a_{i} b} f^{b a_{k} a_{j}} D_{2}(z, \bar{z})\right]\right. \\
&\quad+\{i \leftrightarrow j\}\},
\end{aligned}
$$

with

$$
\begin{aligned}
& D_{1}(z, \bar{z})=u^{-2 \epsilon} F(z, \bar{z})+F\left(\frac{-z}{1-z}, \frac{-\bar{z}}{1-\bar{z}}\right), \\
& D_{2}(z, \bar{z})=u^{-2 \epsilon} F(z, \bar{z})-\left(\frac{u}{v}\right)^{-2 \epsilon}\left[F\left(\frac{1}{z}, \frac{1}{\bar{z}}\right)-F\left(\frac{1-z}{-z}, \frac{1-\bar{z}}{-\bar{z}}\right)\right],
\end{aligned}
$$

and $z=z_{k}^{i j}, 1-z=z_{k}^{j i}$ via the definitions in eqs. (3.8) and (3.9).

\footnotetext{
${ }^{4}$ Occasionally, we suppress the explicit functional dependence on $\bar{z}$. When the explicit dependence is required, e.g. in order to specify a particular analytic continuation where $\bar{z} \neq z^{*}$, we write both arguments. We hope our shortened notation does not cause any confusion.
} 
In order to obtain eq. (3.16) we used the manifest symmetry of $\boldsymbol{S}_{a, i k j}^{+,(2)}$ under exchange of legs $i$ and $j$, the Schouten relation between tree-level eikonal factors, and the Jacobi relation for the gauge-theory structure constants, thereby rearranging the sum into a minimal number of transcendental functions, $D_{1}$ and $D_{2}$. This rearrangement is important because $D_{1}$ and $D_{2}$ should have unambiguous analytic continuations into regions $A_{1}, A_{2}$ and $A_{3}$. (As we will explain shortly, using the symmetry of the tripole formula (3.16), it is sufficient to discuss only the $A_{1}$ discontinuity.) In contrast, the analytic continuation of $F$ has ambiguities that only cancel due to identities among its rational prefactors.

The functions $D_{1}$ and $D_{2}$ can be computed from $F$ in eq. (3.14), after transforming the $\operatorname{arguments}^{5}$ of the SVHPLs back to a uniform argument $(z, \bar{z})$. The results are:

$$
\begin{aligned}
D_{1}(z)= & -\frac{1}{\epsilon^{2}}\left(\mathcal{L}_{1}\right)^{2}-\frac{1}{\epsilon}\left(\mathcal{L}_{1}\right)^{3}-\frac{7}{12}\left(\mathcal{L}_{1}\right)^{4}+4 \mathcal{L}_{1,0,1,0}+2 \mathcal{L}_{1,0,1,1}+2 \mathcal{L}_{1,1,1,0} \\
D_{2}(z)= & \frac{1}{\epsilon^{2}} \mathcal{L}_{0} \mathcal{L}_{1}+\frac{1}{\epsilon} \mathcal{L}_{0}\left(\mathcal{L}_{1}\right)^{2}+\frac{2}{3} \mathcal{L}_{0}\left(\mathcal{L}_{1}\right)^{3}+6 \zeta_{2}\left(\mathcal{L}_{0,1}-\mathcal{L}_{1,0}\right) \\
& +2\left(\mathcal{L}_{0,0,0,1}-\mathcal{L}_{0,0,1,0}+\mathcal{L}_{0,1,0,0}+\mathcal{L}_{0,1,0,1}-\mathcal{L}_{1,0,0,0}\right)
\end{aligned}
$$

For the $i \leftrightarrow j$ term in eq. (3.16), it is convenient to have the same functions with argument $1-z$, expressed in terms of SVHPLs with argument $z$ :

$$
\begin{aligned}
D_{1}(1-z)= & -\frac{1}{\epsilon^{2}}\left(\mathcal{L}_{0}\right)^{2}+\frac{1}{\epsilon}\left(\mathcal{L}_{0}\right)^{3}-\frac{7}{12}\left(\mathcal{L}_{0}\right)^{4}+4 \mathcal{L}_{0,1,0,1}+2 \mathcal{L}_{0,1,0,0}+2 \mathcal{L}_{0,0,0,1} \\
& +8 \zeta_{3} \mathcal{L}_{0} \\
D_{2}(1-z)= & \frac{1}{\epsilon^{2}} \mathcal{L}_{0} \mathcal{L}_{1}-\frac{1}{\epsilon}\left(\mathcal{L}_{0}\right)^{2} \mathcal{L}_{1}+\frac{2}{3}\left(\mathcal{L}_{0}\right)^{3} \mathcal{L}_{1}+6 \zeta_{2}\left(\mathcal{L}_{1,0}-\mathcal{L}_{0,1}\right)-8 \zeta_{3} \mathcal{L}_{1} \\
& +2\left(\mathcal{L}_{1,1,1,0}-\mathcal{L}_{1,1,0,1}+\mathcal{L}_{1,0,1,1}+\mathcal{L}_{1,0,1,0}-\mathcal{L}_{0,1,1,1}\right)
\end{aligned}
$$

It is possible to write $D_{1}$ and $D_{2}$ explicitly in terms of classical polylogarithms. However, the results are not particularly compact, and the single-valuedness is not particularly manifest:

$$
\begin{aligned}
D_{1}(z)= & -\frac{\log ^{2}|1-z|^{2}}{\epsilon^{2}}+\frac{\log ^{3}|1-z|^{2}}{\epsilon} \\
& +8\left[\operatorname{Li}_{4}(z)-\operatorname{Li}_{4}(\bar{z})+\operatorname{Li}_{4}\left(\frac{-z}{1-z}\right)-\operatorname{Li}_{4}\left(\frac{-\bar{z}}{1-\bar{z}}\right)\right] \\
& +4\left(\log |1-z|^{2}-2 \log |z|^{2}\right)\left[\operatorname{Li}_{3}(z)+\operatorname{Li}_{3}\left(\frac{-z}{1-z}\right)\right] \\
& +4 \log |1-z|^{2}\left[\operatorname{Li}_{3}(\bar{z})-\operatorname{Li}_{3}\left(\frac{-\bar{z}}{1-\bar{z}}\right)\right]+2\left(\operatorname{Li}_{2}(z)-\operatorname{Li}_{2}(\bar{z})\right)^{2} \\
& +\left(4 \log (1-z) \log |z|^{2}-\log ^{2}|1-z|^{2}\right)\left(\operatorname{Li}_{2}(z)-\operatorname{Li}_{2}(\bar{z})\right) \\
& +\log (1-z) \log \left(\frac{1-z}{1-\bar{z}}\right) \log |z|^{2} \log |1-z|^{2} \\
& -\frac{1}{12}(11 \log (1-z)+3 \log (1-\bar{z})) \log ^{3}|1-z|^{2}
\end{aligned}
$$

\footnotetext{
${ }^{5}$ In PolyLogTools [65] this can be achieved with the "ToFibrationBasis" command.
} 


$$
\begin{aligned}
D_{2}(z)= & -\frac{\log |z|^{2} \log |1-z|^{2}}{\epsilon^{2}}+\frac{\log |z|^{2} \log ^{2}|1-z|^{2}}{\epsilon} \\
& +4\left\{3\left(\operatorname{Li}_{4}(z)-\operatorname{Li}_{4}(\bar{z})\right)-\operatorname{Li}_{4}\left(\frac{-z}{1-z}\right)+\operatorname{Li}_{4}\left(\frac{-\bar{z}}{1-\bar{z}}\right)\right. \\
& \quad+\operatorname{Li}_{4}(1-z)-\operatorname{Li}_{4}(1-\bar{z})+\left(\log |1-z|^{2}-2 \log |z|^{2}\right) \operatorname{Li}_{3}(z) \\
& \left.\quad+\log |z|^{2}\left[\operatorname{Li}_{3}(\bar{z})-\operatorname{Li}_{3}\left(\frac{-\bar{z}}{1-\bar{z}}\right)\right]\right\} \\
& +\left(\operatorname{Li}_{2}(z)-\operatorname{Li}_{2}(\bar{z})\right)^{2}+2 \log |z|^{2}\left(\log |z|^{2}-\log (1-\bar{z})\right)\left(\operatorname{Li}_{2}(z)-\operatorname{Li}_{2}(\bar{z})\right) \\
& -\frac{1}{12} \log |1-z|^{2} \log ^{3}\left(\frac{1-z}{1-\bar{z}}\right)+\frac{1}{2} \log z \log |1-z|^{2} \log ^{2}\left(\frac{1-z}{1-\bar{z}}\right) \\
& +\frac{1}{3} \log { }^{3}|z|^{2} \log _{(}\left(\frac{1-z}{1-\bar{z}}\right)+\frac{1}{12}\left[\log \left(\frac{z}{\bar{z}}\right)-\log \left(\frac{1-z}{1-\bar{z}}\right)\right] \log ^{3}|1-z|^{2} \\
& -\frac{7}{12} \log |z|^{2} \log ^{3}|1-z|^{2} \\
& +\zeta_{2}\left\{6\left[2\left(\operatorname{Li}_{2}(z)-\operatorname{Li}_{2}(\bar{z})\right)+\log |z|^{2} \log \left(\frac{1-z}{1-\bar{z}}\right)\right]\right. \\
& \left.\quad-2\left(\log ^{2}(1-z)-\log ^{2}(1-\bar{z})\right)\right\} \\
& -4 \zeta_{3} \log |1-z|^{2} .
\end{aligned}
$$

These versions are valid for $0<\operatorname{Re} z<1$, although they can also be extended outside this range. Note that in order to evaluate $\log \left(\frac{1-z}{1-\bar{z}}\right)$ numerically, depending on the computer algebra system, one might have to write this as $\log (1-z)-\log (1-\bar{z})$. For example, Mathematica always assigns a phase between $-\pi$ and $+\pi$ to $(1-z) /(1-\bar{z})$, which can be different from the actual phase.

For complex $z$, the $D_{i}$ functions are generically complex. On the Euclidean sheet, they obey a reality condition: when $z$ is complex conjugated, the functions get complex conjugated,

$$
D_{i}(\bar{z}, z)=\overline{D_{i}(z, \bar{z})}
$$

Interestingly, the imaginary part of $D_{1}(z, \bar{z})$ vanishes identically on the circle of radius 1 centered at $z=1$, which has $|z|^{2}=2 \operatorname{Re} z$, or $\bar{z}=-z /(1-z)$. One can see manifestly that the $\mathrm{Li}_{4}$ terms in eq. (3.23) vanish on this circle, and using $\log |1-z|^{2}=0$ it is easy to see that the rest of the terms are real. The same is not true for $D_{2}$. 
In the case that $z$ is real (when the volume element of $p_{i}, p_{j}, p_{k}$ and $q$ vanishes), $D_{1}$ and $D_{2}$ simplify considerably, to

$$
\begin{aligned}
D_{1}(\bar{z}=z)= & -\frac{4}{\epsilon^{2}} \log ^{2}|1-z|+\frac{8}{\epsilon} \log ^{3}|1-z|-\frac{28}{3} \log ^{4}|1-z| \\
& -16 \log \left|\frac{z}{1-z}\right| \operatorname{Li}_{3}(z)-16 \log |z| \operatorname{Li}_{3}\left(\frac{-z}{1-z}\right) \\
D_{2}(\bar{z}=z)= & -\frac{4}{\epsilon^{2}} \log |z| \log |1-z|+\frac{8}{\epsilon} \log |z| \log ^{2}|1-z|-\frac{28}{3} \log |z| \log ^{3}|1-z| \\
& -8 \log \left|\frac{z}{1-z}\right| \operatorname{Li}_{3}(z)-8 \log |z| \operatorname{Li}_{3}\left(\frac{-z}{1-z}\right)-8 \zeta_{3} \log |1-z|
\end{aligned}
$$

where for $z>1$ one should replace

$$
\begin{aligned}
\operatorname{Li}_{3}(z) \rightarrow & \operatorname{Li}_{3}\left(\frac{1}{z}\right)-\frac{1}{6} \log ^{3} z+2 \zeta_{2} \log z \\
\operatorname{Li}_{3}\left(\frac{-z}{1-z}\right) \rightarrow & -\operatorname{Li}_{3}(1-z)-\operatorname{Li}_{3}\left(\frac{1}{z}\right)+\frac{1}{6} \log ^{3} z+\frac{1}{6} \log ^{3}(z-1) \\
& -\frac{1}{2} \log z \log ^{2}(z-1)+\zeta_{2} \log \left(\frac{z-1}{z^{2}}\right)+\zeta_{3},
\end{aligned}
$$

so that the expressions remain manifestly real. While eqs. (3.26) and (3.27) contain only trilogarithms $\left(\mathrm{Li}_{3}\right)$ and lower-weight functions, it is clear from eqs. (3.23) and (3.24) that the parts of $D_{1}$ and $D_{2}$ that are odd under $z \leftrightarrow \bar{z}$ contain $\mathrm{Li}_{4}$ as well, but those parts vanish on the real line $z=\bar{z}$. It is also interesting that the polylogarithmic parts of $D_{1}$ and $2 D_{2}$ are identical on the real axis.

\subsection{Analytic continuation}

So far we have restricted our discussion to the $A_{0}$ region. To derive phenomenologically relevant results, it is necessary to perform an analytic continuation of the soft factor. The analytic continuation of the dipole terms is trivial and is completely specified by eqs. (2.16) and (2.17). However, the analytic continuation for the tripole contribution is more involved. If zero, two, or three hard legs are outgoing (in addition to the outgoing soft gluon $q$ ), table 1 shows that no analytic continuation from $A_{0}$ is required for either $F$ or $D_{i}$. (Note that the $V_{i j}^{q}$ prefactors can acquire phases from eq. (2.17).) In the $A_{0}$ region, the functions $F$ and $D_{i}$ are real on the real axis and they complex conjugate when $z$ does; see eq. (3.25). As mentioned before, all cases where $q$ is incoming can be obtained from the ones in table 1 by exchanging all incoming and all outgoing momenta.

For the three Minkowski regions $A_{1}, A_{2}$ and $A_{3}$ described in table 1 , the analytic continuation of the tripole emission term is nontrivial. However, using the symmetric tripole formula $\boldsymbol{S}_{a,\{i, j, k\}}^{+,(2)}$, eq. (3.16), it suffices to give the $A_{1}$ discontinuity. In order to see this, note that the tripole $\{i, j, k\}$ is unordered. Therefore, if the soft momentum $q$ is outgoing and exactly one of the three hard legs in the tripole is outgoing, we label that leg by $i$. According to table 1 , such a tripole should be evaluated in region $A_{1}$, which we direct our attention to in the following. 
The difficulty in the analytic continuation of the tripole terms comes from the fact that $z_{k}^{i j}$ and $\bar{z}_{k}^{i j}$ contain square roots of $u_{k}^{i j}$ and $v_{k}^{i j}$, see the quadratic relations in eq. (3.7). To determine the analytic continuation of the SVHPLs entering the $D_{i}$ functions, we use a bottom-up approach (in the sense of transcendental weight). We focus on the tripole labelled by $\{i, j, k\}$ and drop the particle indices $i, j, k$ for now. At weight 1 there are only two SVHPLs, due to the first-entry condition mentioned above:

$$
\mathcal{L}_{0}(z)=\log (z \bar{z})=\log v, \quad \mathcal{L}_{1}(z)=-\log [(1-z)(1-\bar{z})]=-\log u,
$$

whose analytic continuation properties are specified by eq. (2.17) and are summarized in table 1. Starting from weight 1 , we can build the analytic continuation for weight 2 SVHPLs by requiring consistency with the differential equations. As an example, consider $\mathcal{L}_{0,1}(z)$, which in terms of logarithms and polylogarithms is given by

$$
\mathcal{L}_{0,1}(z)=-\log (1-\bar{z}) \log (z \bar{z})+\operatorname{Li}_{2}(z)-\operatorname{Li}_{2}(\bar{z}) .
$$

While it is not so difficult to compute the discontinuity of $\mathcal{L}_{0,1}(z)$ directly, it is even easier to compute the discontinuity of its derivative:

$$
\partial_{z} \mathcal{L}_{0,1}(z)=\frac{\mathcal{L}_{1}(z)}{z}, \quad \partial_{\bar{z}} \mathcal{L}_{0,1}(z)=\frac{\mathcal{L}_{0}(z)}{1-\bar{z}}
$$

For a given region, we first tabulate the discontinuities of $\mathcal{L}_{0}$ and $\mathcal{L}_{1}$. We then use the fact that the operations of taking the discontinuity and taking the derivative commute. We take the discontinuity of eq. (3.32) and then integrate up the right-hand-side, to get the discontinuity of $\mathcal{L}_{0,1}(z)$, up to an additive constant. To determine the constant, we can work near the point where the analytic continuation is being performed. There, the function can only involve logarithms plus irrelevant power corrections and it is easy to analytically continue.

For example, suppose we want to continue all the functions into the $A_{1}$ region. We can deform $z \rightarrow z e^{-2 \pi \mathrm{i}}$ around the origin, keeping $\bar{z}$ constant. ${ }^{6}$ The discontinuities in $\mathcal{L}_{0}$ and $\mathcal{L}_{1}$ are

$$
\begin{aligned}
& \underset{z \rightarrow z e^{-2 \pi \mathrm{i}}}{\operatorname{disc}_{z}}\left[\mathcal{L}_{0}(z, \bar{z})\right] \equiv\left[\mathcal{L}_{0}\left(z e^{-2 \pi \mathrm{i}}, \bar{z}\right)-\mathcal{L}_{0}(z, \bar{z})\right]=-2 \mathrm{i} \pi \\
& \underset{z \rightarrow z e^{-2 \pi \mathrm{i}}}{\operatorname{disc}_{1}}\left[\mathcal{L}_{1}(z, \bar{z})\right] \equiv\left[\mathcal{L}_{1}\left(z e^{-2 \pi \mathrm{i}}, \bar{z}\right)-\mathcal{L}_{1}(z, \bar{z})\right]=0
\end{aligned}
$$

where we have explicitly written two arguments in the (poly)logarithms to indicate that we do not enforce $\bar{z}=z^{*}$ in the analytic continuation. Now we plug eq. (3.33) into the discontinuity of eq. (3.32) and integrate up,

$$
\operatorname{disc}_{z \rightarrow z e^{-2 \pi \mathrm{i}}}\left[\mathcal{L}_{0,1}(z, \bar{z})\right]=2 \mathrm{i} \pi \log (1-\bar{z}) .
$$

The constant can be fixed at $z=\bar{z}=0$, where $\mathcal{L}_{0,1} \rightarrow 0 \times \log (z \bar{z})$, and so the discontinuity must vanish at that point. Note that the discontinuity is no longer a single-valued function.

\footnotetext{
${ }^{6}$ Since $\mathcal{L}_{0,1}(z)$ is a real-analytic function, we can freely split the analytic continuation in terms of $z$ and $\bar{z}$. Equivalently, we could have chosen the symmetric deformation $z \rightarrow z e^{-\mathrm{i} \pi}, \bar{z} \rightarrow \bar{z} e^{-\mathrm{i} \pi}$.
} 
Using this bottom-up approach we can determine the discontinuity (and therefore the analytic continuation) of not just $\mathcal{L}_{0,1}$ but of all the SVHPLs, iteratively to higher weight.

We shall provide the discontinuities $\operatorname{disc}_{A_{1}} D_{i}$ of the functions $D_{i}$ to go from region $A_{0}$ to regions $A_{1}$. The function itself is given by

$$
\left.D_{i}(z, \bar{z})\right|_{A_{1}}=\left.D_{i}(z, \bar{z})\right|_{A_{0}}+\operatorname{disc}_{A_{1}} D_{i}(z, \bar{z}),
$$

where

$$
\operatorname{disc}_{A_{1}} D_{i}(z, \bar{z})=\operatorname{disc}_{z \rightarrow z e^{-2 \pi \mathrm{i}}}\left[D_{i}(z, \bar{z})\right] .
$$

The result for the discontinuity needed to move $D_{1}(z)$ and $D_{2}(z)$ from region $A_{0}$ to region $A_{1}$ can be expressed in terms of classical polylogarithms:

$$
\begin{aligned}
\operatorname{disc}_{A_{1}} D_{1}(z)=2 \mathrm{i} \pi\{ & 8\left[\operatorname{Li}_{3}(z)+\operatorname{Li}_{3}\left(\frac{-z}{1-z}\right)\right] \\
& \left.-\log (1-z)\left[4\left(\operatorname{Li}_{2}(z)-\operatorname{Li}_{2}(\bar{z})\right)+\log ^{2}(1-z)-\log ^{2}(1-\bar{z})\right]\right\} .
\end{aligned}
$$

In this form, $\operatorname{disc}_{A_{1}} D_{1}(z)$ manifestly has no branch cuts for $\operatorname{Re} z<1$. For $\operatorname{Re} z>1$ it is not manifest, but one can check that as $z$ approaches the real axis, the imaginary part in $\operatorname{disc}_{A_{1}}\left[D_{1}(z) /(2 \mathrm{i} \pi)\right]$ cancels, and the result is unambiguous, and equal to:

$$
\begin{aligned}
\left.\operatorname{disc}_{A_{1}} D_{1}(z)\right|_{z>1, \text { real }}=2 \mathrm{i} \pi \times 8\{ & -\operatorname{Li}_{3}(1-z)-\frac{1}{2} \log z\left(\log ^{2}(z-1)+\pi^{2}\right) \\
& \left.+\frac{1}{6} \log ^{3}(z-1)+\zeta_{2} \log (z-1)+\zeta_{3}\right\} .
\end{aligned}
$$

Similarly, the $A_{1}$ discontinuity of the $D_{2}(z)$ function is

$$
\begin{aligned}
\operatorname{disc}_{A_{1}} D_{2}(z)=2 \mathrm{i} \pi\{ & \frac{\log |1-z|^{2}}{\epsilon^{2}}-\frac{\log ^{2}|1-z|^{2}}{\epsilon}+8 \operatorname{Li}_{3}(z)-4 \operatorname{Li}_{3}(\bar{z})+4 \operatorname{Li}_{3}\left(\frac{-\bar{z}}{1-\bar{z}}\right) \\
& -2\left[\operatorname{Li}_{2}(z)-\operatorname{Li}_{2}(\bar{z})\right]\left[2 \log |z|^{2}-\log (1-\bar{z})\right]+2 \zeta_{2} \log \left(\frac{1-z}{1-\bar{z}}\right) \\
& -\log \left(\frac{1-z}{1-\bar{z}}\right) \log ^{2}|z|^{2}+2 \log (1-z) \log (1-\bar{z}) \log |1-z|^{2} \\
& \left.+\frac{2}{3} \log ^{3}(1-z)\right\} \\
- & 4 \pi^{2}\left\{2\left[\operatorname{Li}_{2}(z)-\operatorname{Li}_{2}(\bar{z})\right]+\log \left(\frac{1-z}{1-\bar{z}}\right) \log |z|^{2}\right\} .
\end{aligned}
$$

This result also has a well-defined limit in the region where $z=\bar{z} \in(1, \infty)$,

$$
\begin{aligned}
\left.\operatorname{disc}_{A_{1}} D_{2}(z)\right|_{z>1, \text { real }}=4 \mathrm{i} \pi & \frac{\log (z-1)}{\epsilon^{2}}-\frac{2 \log ^{2}(z-1)}{\epsilon}-2 \operatorname{Li}_{3}(1-z)+\frac{8}{3} \log ^{3}(z-1) \\
& \left.-\log z\left(\log ^{2}(z-1)+\pi^{2}\right)+2 \zeta_{2} \log (z-1)+2 \zeta_{3}\right\}
\end{aligned}
$$


We also need the $A_{1}$ discontinuity of $D_{1}(1-z)$,

$$
\begin{aligned}
\operatorname{disc}_{A_{1}} D_{1}(1-z)=2 \mathrm{i} \pi\{ & \frac{2 \log |z|^{2}}{\epsilon^{2}}-\frac{3}{\epsilon}\left(\log ^{2}|z|^{2}-8 \zeta_{2}\right)+4 \operatorname{Li}_{3}(z)+4 \operatorname{Li}_{3}(\bar{z}) \\
& +8 \operatorname{Li}_{3}\left(\frac{-\bar{z}}{1-\bar{z}}\right)-2\left[\operatorname{Li}_{2}(z)-\operatorname{Li}_{2}(\bar{z})\right]\left[\log |z|^{2}-2 \log (1-\bar{z})\right] \\
& -\frac{4}{3} \log ^{3}(1-\bar{z})+\log (1-\bar{z}) \log ^{2}|z|^{2}+\frac{7}{3} \log ^{3}|z|^{2} \\
& \left.-8 \zeta_{2}\left[7 \log |z|^{2}+\log (1-\bar{z})\right]-8 \zeta_{3}\right\} \\
-4 \pi^{2}\{ & -\frac{1}{\epsilon^{2}}+\frac{3}{\epsilon} \log |z|^{2}-\frac{7}{2} \log ^{2}|z|^{2}+\operatorname{Li}_{2}(z)-\operatorname{Li}_{2}(\bar{z}) \\
& \left.-\log (1-\bar{z}) \log |z|^{2}+14 \zeta_{2}\right\}
\end{aligned}
$$

For $z=\bar{z} \in(1, \infty)$ it becomes

$$
\begin{aligned}
\left.\operatorname{disc}_{A_{1}} D_{1}(1-z)\right|_{z>1, \text { real }}=2 \mathrm{i} & \left\{\frac{4 \log z}{\epsilon^{2}}-\frac{12}{\epsilon}\left[\log ^{2} z-2 \zeta_{2}\right]\right. \\
& +8\left[-\operatorname{Li}_{3}(1-z)+\frac{7}{3} \log ^{3} z+\frac{1}{2} \log ^{2} z \log (z-1)\right. \\
& \left.\left.-\frac{1}{2} \log z \log ^{2}(z-1)-17 \zeta_{2} \log z\right]\right\} \\
-4 \pi^{2}\{ & -\frac{1}{\epsilon^{2}}+\frac{6 \log z}{\epsilon}-14\left(\log ^{2} z-\zeta_{2}\right) \\
& -2 \log z \log (z-1)\}
\end{aligned}
$$

Finally, the $A_{1}$ discontinuity of $D_{2}(1-z)$ is

$$
\begin{aligned}
\operatorname{disc}_{A_{1}} D_{2}(1-z)= & 2 \mathrm{i} \pi\left\{\frac{\log |1-z|^{2}}{\epsilon^{2}}-\frac{2}{\epsilon} \log |z|^{2} \log |1-z|^{2}+4 \operatorname{Li}_{3}(z)+4 \operatorname{Li}_{3}\left(\frac{-z}{1-z}\right)\right. \\
& -2 \log (1-z)\left[\operatorname{Li}_{2}(z)-\operatorname{Li}_{2}(\bar{z})-\log ^{2}(1-\bar{z})-6 \zeta_{2}\right] \\
& \left.\quad-\frac{1}{3} \log ^{3}|1-z|^{2}+2 \log ^{2}|z|^{2} \log |1-z|^{2}-22 \zeta_{2} \log |1-z|^{2}\right\} \\
& -4 \pi^{2}\left\{\frac{\log |1-z|^{2}}{\epsilon}-2 \log |z|^{2} \log |1-z|^{2}\right\} \\
= & \frac{1}{2} \operatorname{disc}_{A_{1}} D_{1}(z)-4 \pi^{2}\left\{\frac{\log |1-z|^{2}}{\epsilon}-2 \log |z|^{2} \log |1-z|^{2}\right\} \\
& +2 \mathrm{i} \pi\left\{\frac{\log |1-z|^{2}}{\epsilon^{2}}-\frac{2}{\epsilon} \log |z|^{2} \log |1-z|^{2}-\frac{1}{12} \log ^{3}|1-z|^{2}\right. \\
& \quad+2 \log |z|^{2} \log |1-z|^{2}-16 \zeta_{2} \log |1-z|^{2} \\
& \left.\quad+\frac{1}{4} \log ^{2}\left(\frac{1-z}{1-\bar{z}}\right)\left[\log ^{2}\left(\frac{1-z}{1-\bar{z}}\right)+4 \pi^{2}\right]\right\}
\end{aligned}
$$


The second form (3.44) shows that the linear combination $D_{2}(1-z)-\frac{1}{2} D_{1}(z)$ has a discontinuity that is purely logarithmic. It illustrates more simply the unambiguous behavior as $z$ approaches the real axis with $\operatorname{Re}(z)>1$. The last term in eq. (3.44) is the only one with a potential ambiguity, but it cancels because $\log \left(\frac{1-z}{1-\bar{z}}\right) \rightarrow 2 \mathrm{i} \pi$, and $(2 \mathrm{i} \pi)^{2}+4 \pi^{2}=0$. In this limit, $D_{2}(1-z)$ becomes

$$
\begin{aligned}
\left.\operatorname{disc}_{A_{1}} D_{2}(1-z)\right|_{z>1, \text { real }}=2 \mathrm{i} \pi & \left\{\frac{2 \log (z-1)}{\epsilon^{2}}-\frac{8 \log z \log (z-1)}{\epsilon}\right. \\
& -4\left(\operatorname{Li}_{3}(1-z)-\zeta_{3}\right)-2 \log z\left[\log ^{2}(z-1)+\pi^{2}\right] \\
& \left.+4\left[4 \log ^{2} z-7 \zeta_{2}\right] \log (z-1)\right\} \\
-4 \pi^{2} & \left\{\frac{2 \log (z-1)}{\epsilon}-8 \log z \log (z-1)\right\} .
\end{aligned}
$$

To summarize, we combined the tripole contributions to the two-loop soft factor into four sets of SVHPLs: $\left\{D_{i}(z), D_{i}(1-z)\right\}, i=\{1,2\}$, given in eqs. (3.19)-(3.22). Each of the $D_{i}$ 's can be defined in either region $A_{0}$, or in $A_{1}$ via analytic continuation. The other regions are not needed explicitly, due to the freedom in labeling the unordered tripole $\{i, j, k\}$. After analytic continuation, the $D_{i}$ are not single-valued functions, in the sense that they cannot be written in terms of the $\mathcal{L}_{\vec{w}}$ functions, but they are unambiguously defined in the physical domain where $\bar{z}$ is the complex conjugate of $z$; that is, where $\Delta=z-\bar{z}$ is purely imaginary, i.e. $1-2 u-2 v+(u-v)^{2} \leq 0$. We emphasize that this is a nontrivial property, which is not satisfied by the discontinuities of arbitrary linear combinations of SVHPLs.

For negative-helicity emission, one should swap $z \leftrightarrow \bar{z}$ everywhere, as well as letting $\langle a b\rangle \leftrightarrow[b a]$ in the rational prefactors, but the manifest 'i's in the discontinuity formulas should not be flipped. The results for the $D_{i}$ functions in region $A_{0}$, their symbols, and their discontinuities for the analytic continuation into the $A_{1}$ region, are all included in the supplementary material attached to this paper.

\section{Applications}

\subsection{Soft limits of scattering amplitudes}

The two-loop soft factor predicts the soft-gluon limit of a generic two-loop $n$-point scattering amplitude, when the corresponding $(n-1)$-point amplitude without the soft gluon is known. Such limits can provide stringent checks of two-loop amplitudes, starting at five points. The tripole terms contribute to amplitudes beyond the planar limit, which are only just beginning to become available at five points.

As an example, we can construct the soft limit of the full color two-loop five-gluon maximally helicity violating (MHV) amplitude in $\mathcal{N}=4 \mathrm{SYM}[31,32]$, using the well- 
known full color two-loop four-gluon MHV amplitude [66-68],

$$
\begin{aligned}
\lim _{q^{\mu} \rightarrow 0} & \left|\mathcal{M}_{5}^{\mathrm{MHV},(2)}\left(1^{-}, 2^{-}, 3^{+}, 4^{+}, q^{+}\right)\right\rangle \\
& =\sum_{\ell=0}^{2} \boldsymbol{S}^{+,(\ell)}\left(\left\{\beta_{m}\right\}\right)\left|\mathcal{M}_{4}^{\mathrm{MHV},(2-\ell)}\left(1^{-}, 2^{-}, 3^{+}, 4^{+}\right)\right\rangle .
\end{aligned}
$$

The $1 / \epsilon$ infrared singularities on both sides of eq. (4.1) agree with those obtained from the so-called dipole formula [39-42, 69, 70], which provides a strong check of our result. Furthermore, at $\mathcal{O}\left(\epsilon^{0}\right)$, we have evaluated both sides explicitly in the trace basis for five external gluons, and the symbol [60] of our soft-emission formula matches perfectly the soft limit of the symbol-level results for the two-loop five-particle scattering amplitudes in $\mathcal{N}=4$ SYM [31, 32]. We anticipate that the knowledge of the soft factor provided here at function level will play an important role in fixing certain beyond-the-symbol constants in the supersymmetric amplitude, as well as for amplitudes with less supersymmetry in the future.

\subsection{Soft-collinear limit}

As a further interesting application of our result, we can derive the soft-gluon limit of the two-loop collinear splitting amplitudes for $g \rightarrow g g, q \rightarrow q g$, or $\bar{q} \rightarrow \bar{q} g$ from the two-loop soft factor computed above. In the case of timelike splitting, where an off-shell parton splits into two on-shell partons in the final state, the two-loop splitting amplitudes have been computed by several groups [26, 47, 71, 72].

However, for splitting amplitudes with spacelike kinematics, two-loop splitting amplitudes are so far unknown except for their singular pieces [33]. Before we discuss the soft limit for both timelike and spacelike splitting at two loops, let us briefly comment on the relevant collinear kinematic setup, establish our conventions, and discuss the collinear limit of the tree-level and one-loop soft factors.

To be specific, consider an $(n+1)$-point scattering process, where $q$ is the momentum of an outgoing soft gluon which is collinear to $p_{1}$. We distinguish two cases:

- timelike splitting: particle 1 is an outgoing parton with momentum $p_{1}$,

- spacelike splitting: particle 1 is an incoming parton with momentum $-p_{1}$

(in all outgoing conventions).

Let us define the longitudinal momentum fraction $x_{q}$ carried by the soft gluon with respect to the parent parton $P$, such that

$$
q \rightarrow x_{q} P, \quad p_{1} \rightarrow\left(1-x_{q}\right) P, \quad \text { where } \quad P^{\mu} \equiv q^{\mu}+p_{1}^{\mu}-\frac{\left(q \cdot p_{1}\right) n^{\mu}}{\left(q+p_{1}\right) \cdot n}
$$

is a lightlike momentum, $P^{2}=0$, and $n^{\mu}$ defines an auxiliary lightlike vector. In the case where $q$ is soft, $P \cong p_{1}$, so that $\left|x_{q}\right| \ll 1$. Depending on whether we consider timelike or spacelike splitting, the sign of $x_{q}$ changes, from $x_{q}>0$ to $x_{q}<0$, respectively. 
Before discussing the loop-level analysis of the soft-collinear limit, let us consider the collinear limit of the tree-level soft-factor $\boldsymbol{S}_{a}^{+,(0)}$ in eq. (2.13),

$$
\boldsymbol{S}_{a}^{+,(0)}=\frac{1}{2 n} \sum_{i \neq j}\left(\boldsymbol{T}_{i}^{a_{i}}-\boldsymbol{T}_{j}^{a_{j}}\right) \frac{\langle i j\rangle}{\langle i q\rangle\langle q j\rangle}=\frac{1}{n} \sum_{j<i}\left(\boldsymbol{T}_{i}^{a_{i}}-\boldsymbol{T}_{j}^{a_{j}}\right) \frac{\langle i j\rangle}{\langle i q\rangle\langle q j\rangle} .
$$

We would like to consider the (timelike) collinear limit $q \| p_{1}$ (or $q \| 1$ for short). The only terms containing the singular factor $\langle q 1\rangle$ are those with $j=1$,

$$
\lim _{q \| p_{1}}\left[\boldsymbol{S}_{a}^{+,(0)}\right]=\frac{1}{n} \sum_{i=2}^{n}\left(\boldsymbol{T}_{i}^{a_{i}}-\boldsymbol{T}_{1}^{a_{1}}\right) \frac{\langle i 1\rangle}{\langle i q\rangle\langle q 1\rangle} .
$$

In the collinear limit (with $q$ also soft), we can now use,

$$
\lambda_{1} \simeq \sqrt{1-x_{q}} \lambda_{P} \simeq \lambda_{P}, \quad \lambda_{q} \simeq \sqrt{x_{q}} \lambda_{P} \quad \Rightarrow \quad \frac{\langle i 1\rangle}{\langle i q\rangle\langle q 1\rangle} \simeq \frac{1}{\sqrt{x_{q}}\langle q 1\rangle},
$$

which is independent of $i$, so that we can use color conservation $\sum_{i=2}^{n} \boldsymbol{T}_{i}^{a_{i}}=-\boldsymbol{T}_{1}^{a_{1}}$ in the first term of (4.4). In the second term, we get $(n-1)$ times the same contribution, which combined with the first term cancels the factor of $1 / n$. We finally obtain the $q \| 1$ collinear limit of the tree-level soft factor,

$$
\lim _{q \| p_{1}}\left[\boldsymbol{S}_{a}^{+,(0)}\right]=\boldsymbol{T}_{1}^{a_{1}} \frac{1}{\sqrt{x_{q}}\langle 1 q\rangle} \equiv \mathbf{S p}^{(0)} .
$$

Note that the full collinear splitting function depends on the helicities of the collinear partons, see e.g. ref. [71] (there it is denoted by $\mathbf{S p l i t}_{-\lambda}^{(0)}$ ); the helicity of $P$ is indicated by the helicity label $\lambda$. (For a summary of tree-level helicity splitting amplitudes, see e.g. ref. [5] or appendix A of ref. [9].) Here, however, we are considering the soft limit of a positive helicity gluon, and the helicity information of the collinear splitting drops out, in the sense that the splitting amplitudes either vanish (due to helicity conservation rules) or become the same function. Thus we drop the helicity label on $\mathbf{S p}$ in the following. The same discussion is also applicable for spacelike splittings, which can be obtained easily by crossing at tree level, replacing $x_{q} \rightarrow-x_{q}$ in eq. (4.6).

Next we consider the spacelike collinear limit of the one-loop soft factor (2.14),

$$
\lim _{q \| p_{1}}\left[\boldsymbol{S}_{a}^{+,(1)}\right]=\sum_{k \neq 1} \boldsymbol{T}_{1}^{a_{1}} \frac{1}{\sqrt{-x_{q}}\langle 1 q\rangle}\left(\frac{\mu^{2}}{x_{q} s_{1 q}}\right)^{\epsilon} f_{a a_{1} a_{k}} \boldsymbol{T}_{k}^{a_{k}} \exp \left[(-1)^{\lambda_{k q}+1} \mathrm{i} \pi \epsilon\right] C_{1}(\epsilon) .
$$

Note that $x_{q} s_{1 q}$ is always positive. Following the definition of the color generators in the adjoint representation, we write $\left(T_{q}^{a}\right)_{b c} \equiv-f^{a b c}$. The sum over dipoles give a contribution to the one-loop splitting amplitude,

$$
\mathbf{S p}^{(1)} \stackrel{q-\text { soft }}{\simeq}-\left(\frac{\mu^{2}}{x_{q} s_{1 q}}\right)^{\epsilon} C_{1}(\epsilon) \sum_{k \neq 1} \boldsymbol{T}_{q} \cdot \boldsymbol{T}_{k} \exp \left[(-1)^{\lambda_{k q}+1} \mathrm{i} \pi \epsilon\right] \mathbf{S p}^{(0)}
$$

where $\mathbf{S p}^{(0)}=\boldsymbol{T}_{1} \frac{1}{\sqrt{-x_{q}}\langle 1 q\rangle}$ is the soft limit of the spacelike tree-level splitting amplitude, which is obtained from the timelike splitting amplitude of eq. (4.6) by crossing (including replacing $\left.x_{q} \rightarrow-x_{q}\right)$. 
Note that the one-loop splitting amplitude in eq. (4.8) already violates the so-called strict collinear factorization, which states that the splitting amplitude should only depend on the quantum numbers (color, spin and kinematics) of the parent parton and of the splitting pair [33] and not on any information of the non-collinear partons. This condition is violated at the amplitude level due to the existence of the $\lambda_{k q}$-dependent phase factor in eq. (4.8), which obstructs the use of color conservation that was used in the tree-level case. Fortunately, the $\lambda_{k q}$ dependence is purely imaginary and cancels out for cross-section level quantities at this perturbative order, which involve only the combination $\mathbf{S p}^{(0)} \mathbf{S} \mathbf{p}^{(1) \dagger}+$ $\mathbf{S} \mathbf{p}^{(0) \dagger} \mathbf{S} \mathbf{p}^{(1)}$. See the discussion around eq. (4.18) for more details.

In the timelike case, the factor $\left(V_{1 k}^{q}\right)^{\epsilon}$, which led to the $k$-dependent phase in the spacelike case in eq. (4.8), behaves differently: the $k$-dependent phases cancel between the numerator and denominator of $\left(V_{1 k}^{q}\right)^{\epsilon}$ in the timelike case.

Soft-collinear analysis for timelike splitting at two loops. For timelike splitting where the outgoing soft gluon with momentum $q$ is collinear to the outgoing particle 1 with momentum $p_{1}$, we have $s_{1 q}>0$ and $x_{q}>0$. The soft limit of the two-loop splitting amplitude can be obtained by taking the collinear limit of the two-loop dipole and tripole contributions, using eqs. (3.1) and (3.15),

$$
\lim _{q \| p_{1}}\left[\boldsymbol{S}_{a}^{+,(2)}\right]=\lim _{q \| p_{1}}\left[\sum_{j \neq 1} \boldsymbol{S}_{a, 1 j}^{+,(2)}-\frac{1}{4} \sum_{\{1, j, k\}} \boldsymbol{S}_{a,\{1, j, k\}}^{+,(2)}\right] .
$$

The collinear limit of the dipole soft-emission term at two loops is straightforward; it is entirely captured by the collinear limit of $\left(V_{1 j}^{q}\right)^{2}$ and the tree-level eikonal factor in eq. (3.2) and therefore we do not discuss it any further for the timelike case.

Due to the nontrivial kinematic dependence, the collinear limit of the tripole softemission term given in eq. (3.16) is more interesting. For timelike splitting kinematics, and assuming at most two partons in the initial state, there are three cases to consider (for an outgoing soft gluon), cf. figures $2 \mathrm{a}, 2 \mathrm{~b}, 2 \mathrm{c}$ :

a) The collinear limit of the tripole term in the $A_{0}$ region, where all hard partons $\{i, j, k\}$ are outgoing and the soft gluon is collinear to either $i, j$ or $k$, see figure $2 \mathrm{a}$.

b) The collinear limit of a tripole with two incoming hard partons and one outgoing hard parton to which the soft gluon is collinear (regions $A_{1,2,3}$, figure $2 \mathrm{~b}$ ).

c) The collinear limit of a tripole with one incoming and three outgoing lines (regions $A_{4,5,6}$, figure 2c) where the outgoing soft gluon is collinear to an outgoing hard parton.

We first discuss the timelike collinear limit of the soft-emission tripole of eq. (3.16) in the $A_{0}$ region of table 1 . In principle, there are three possibilities to take the collinear limit, where the soft gluon $q$ becomes collinear to either of the three hard lines $\{i, j, k\}$ to be identified with $p_{1}$. Since the tripole formula (3.16) is completely symmetric in $\{i, j, k\}$ it suffices to look at a single collinear limit, which we take to be $i=1$ for convenience. Any other implementation of the collinear limit, e.g. with $j=1$ or $k=1$, can then be used as a cross check of our setup. In the $q \| p_{1}\left(=p_{i}\right)$ collinear limit, only the first eikonal factor in eq. (3.16) (and not the $\{i \leftrightarrow j\}$ exchange term) contains the singular denominator $1 /\langle i q\rangle$, 


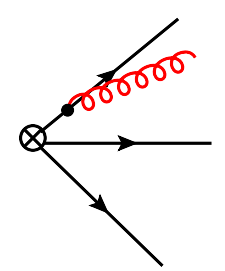

(a)

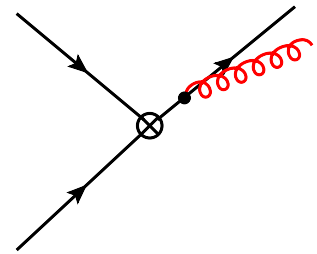

(b)

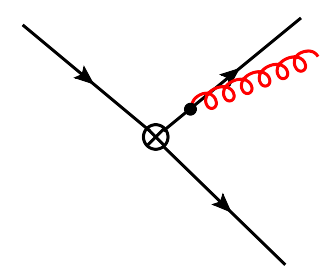

(c)

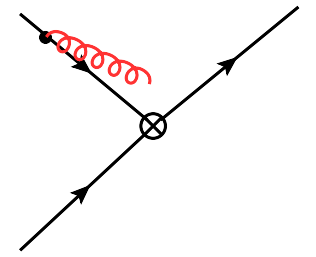

(d)

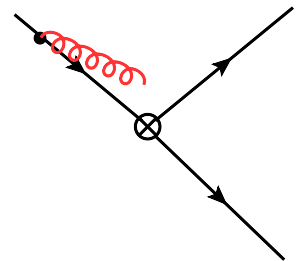

(e)

Figure 2. Kinematics of a tripole contribution to the soft factor in the collinear limit in different regions: (a) timelike splitting in $A_{0}$, (b) timelike splitting in $A_{1}, A_{2}, A_{3}$, (c) timelike splitting in $A_{4}, A_{5}, A_{6}$, (d) spacelike splitting in $A_{1}, A_{2}, A_{3}$, (e) spacelike splitting in $A_{4}, A_{5}, A_{6}$. The curly gluon line represents the outgoing soft gluon with momentum $q$, while the lines with arrows are the tripole legs $i, j, k$. The arrow of time flows from left to right.

so that we need to analyze the limit of the transcendental functions $D_{1}(z, \bar{z})$ and $D_{2}(z, \bar{z})$ in eq. (3.16) as $z=z_{k}^{i j}=\langle k j\rangle\langle i q\rangle /(\langle i j\rangle\langle k q\rangle) \rightarrow 0$ (and likewise $\bar{z} \rightarrow 0$ ). The only SVHPLs that do not vanish at the origin are $\mathcal{L}_{0^{n}}, n=1,2, \ldots$. Since these functions don't appear alone in any term in eqs. (3.19) and (3.20) for $D_{1}$ and $D_{2}$, we find that

$$
\lim _{z, \bar{z} \rightarrow 0} D_{1}(z, \bar{z})=\lim _{z, \bar{z} \rightarrow 0} D_{2}(z, \bar{z})=0
$$

This result can also be seen from the explicit representations of these functions in terms of classical polylogarithms in eqs. (3.23) and (3.24), respectively. Thus the $D_{i}(z, \bar{z})$ provide a power suppression that cancels the leading $1 /\langle i q\rangle$ singularity in the eikonal factor in eq. (3.16).

Due to the manifest tripole symmetry under the $i \leftrightarrow j$ exchange, the $q \| j$ collinear limit $(z \rightarrow 1)$ of the tripole formula is equally suppressed. A little more nontrivially, for consistency of the tripole symmetry, one can also analyze the $q \| k$ collinear limit of (3.16). Here both eikonal factors have the $1 /\langle q k\rangle$ pole, and we wish to send $z, \bar{z} \rightarrow \infty$. However, the kinematic factors become the same in both terms $\left(\lambda_{q} \rightarrow \sqrt{x_{q}} \lambda_{k}, \widetilde{\lambda}_{q} \rightarrow \sqrt{x_{q}} \widetilde{\lambda}_{k}\right)$ :

$$
\begin{gathered}
\frac{\langle i k\rangle}{\langle i q\rangle\langle q k\rangle}\left(V_{i k}^{q}\right)^{2} \stackrel{q \| k}{\longrightarrow} \frac{1}{\sqrt{x_{q}}\langle k q\rangle}\left[\frac{\mu^{2}}{x_{q}\left(-s_{k q}\right)}\right]^{2 \epsilon}, \\
\frac{\langle j k\rangle}{\langle j q\rangle\langle q k\rangle}\left(V_{j k}^{q}\right)^{2} \stackrel{q \| k}{\longrightarrow} \frac{1}{\sqrt{x_{q}}\langle k q\rangle}\left[\frac{\mu^{2}}{x_{q}\left(-s_{k q}\right)}\right]^{2 \epsilon} .
\end{gathered}
$$

Also, the color factors associated with $D_{1}(z)$ and $D_{2}(1-z)$, as well as with $D_{2}(z)$ and $D_{1}(1-z)$, are the same up to a sign. Finally, we find that the $D_{i}$ functions all have the 
same logarithmic divergence as $z \rightarrow \infty$,

$$
\lim _{z \rightarrow \infty} D_{1}(z)=\lim _{z \rightarrow \infty} D_{2}(z)=\lim _{z \rightarrow \infty} D_{1}(1-z)=\lim _{z \rightarrow \infty} D_{2}(1-z) .
$$

Therefore the $q \| k$ collinear limit is also power suppressed as expected. We conclude that in the $A_{0}$ region, the tripole emission contribution is subleading by a power of $q$ when taking the collinear limit inside the soft limit.

The second case to consider is the tripole with two incoming hard partons and one outgoing hard parton to which the soft gluon is collinear, figure $2 \mathrm{~b}$. This collinear limit requires the analysis of the discontinuity of the tripole term to the $A_{1}$ region where we have judiciously chosen the collinear hard parton to be labelled by $i=1$. Looking at the discontinuities of the $D$ functions associated with the eikonal factor that contains the $\langle i q\rangle$ pole, one can indeed check from eqs. (3.37) and (3.39) that these also vanish in the collinear limit $z \rightarrow 0$,

$$
\lim _{z, \bar{z} \rightarrow 0} \operatorname{disc}_{A_{1}} D_{1}(z, \bar{z})=\lim _{z, \bar{z} \rightarrow 0} \operatorname{disc}_{A_{1}} D_{2}(z, \bar{z})=0 .
$$

Finally, since the configurations with two outgoing hard partons (regions $A_{4}, A_{5}$ and $A_{6}$ in table 1 , cf. figure 2c) do not require an analytic continuation, the timelike collinear limits of those tripoles are also suppressed by a power of the soft momentum $q$.

In conclusion, for timelike splitting kinematics, all tripole collinear limits are power suppressed and the leading collinear singular information is provided by the dipole emission contribution. (In the timelike case, the two-loop dipole contribution is also rather innocuous, because there is no $k$-dependent phase, as was mentioned at the end of the previous subsection for the one-loop case.) The subleading nature of tripole emission in the soft-collinear limit is quite reasonable, because the soft-collinear limit of a tripole is conformally equivalent to making the other two hard legs collinear. For example, $q \| p_{i}$ and $p_{j} \| p_{k}$ both send $z_{k}^{i j} \rightarrow 0$. When the two hard partons $p_{j}$ and $p_{k}$ are collinear and both are incoming or both outgoing, the soft gluon cannot resolve the independent colors of the two particles as a consequence of color coherence; it just sees the sum, and therefore the emission is dipole-like. However, in the spacelike case where $p_{j}$ and $p_{k}$ are incoming and outgoing, respectively, this simple picture breaks down. The physical origin of the breakdown of color coherence in this case is related to the Feynman i $\varepsilon$ prescription, and therefore to the causality of the theory. Ultimately, this can lead to a violation of strict factorization, as we shall discuss in the next subsection.

Soft-collinear analysis for spacelike splitting at two loops and strict collinear factorization violation. Let us now discuss spacelike splitting at two loops, where particle 1 is an incoming parton with momentum $-p_{1}$ (in all outgoing conventions) so that $s_{1 q}<0$, and the longitudinal momentum fraction is $x_{q}<0$. First we relabel indices in the two-loop soft-collinear factor (4.9) to obtain

$$
\lim _{q \| p_{1}} \boldsymbol{S}_{a}^{+,(2)}=\lim _{q \| p_{1}}\left[\sum_{k \neq 1} \boldsymbol{S}_{a, 1 k}^{+,(2)}-\frac{1}{4} \sum_{\{i, 1, k\}} \boldsymbol{S}_{a,\{i, 1, k\}}^{+,(2)}\right] .
$$

This labeling will allow us to utilize the $A_{1}$ discontinuity discussed in section 3.3 . 
The analysis of the collinear limit of the dipole term in eq. (4.15) follows closely the one-loop dipole analysis, eq. (4.7). The sum over dipoles gives a contribution to the twoloop collinear splitting amplitude,

$$
\left.\mathbf{S p}^{(2)}\right|_{\text {dipole }} \stackrel{q-\text { soft }}{\simeq}-\left(\frac{\mu^{2}}{x_{q} s_{1 q}}\right)^{2 \epsilon} C_{2}(\epsilon) \sum_{k \neq 1} \boldsymbol{T}_{q} \cdot \boldsymbol{T}_{k} \exp \left[(-1)^{\lambda_{k q}+1} 2 \mathrm{i} \pi \epsilon\right] \mathbf{S p}^{(0)} .
$$

For $L=1$ or 2 we can rewrite the Hermitian part of the dipole terms in eqs. (4.7) and (4.16) by applying color conservation:

$$
\sum_{k \neq 1} \boldsymbol{T}_{q} \cdot \boldsymbol{T}_{k}=-\boldsymbol{T}_{q} \cdot\left(\boldsymbol{T}_{1}+\boldsymbol{T}_{q}\right)=-\frac{1}{2}\left(C_{1 q}-C_{1}\right)-\frac{1}{2} C_{q}=-\frac{1}{2} C_{A},
$$

where $C_{1 q}, C_{1}\left(=C_{1 q}\right)$, and $C_{q}=C_{A}$ are the Casimir coefficients of parton $P$, parton 1 , and the soft-collinear gluon, respectively. The result is

$$
\left.\mathbf{S p}^{(L)}\right|_{\text {dipole }} \stackrel{q-\text { soft }}{\simeq}\left(\frac{\mu^{2}}{x_{q} s_{1 q}}\right)^{L \epsilon} C_{L}(\epsilon)\left\{i \sin (L \pi \epsilon) \sum_{k \neq 1}(-1)^{\lambda_{k q}} \boldsymbol{T}_{q} \cdot \boldsymbol{T}_{k}+\frac{C_{A}}{2} \cos (L \pi \epsilon)\right\} \mathbf{S p}^{(0)}
$$

The factorization-violating kinematic dependence of the $L$-loop dipole term is antiHermitian. The Hermitian part proportional to $\cos (L \pi \epsilon)$ strictly factorizes.

We turn now to the tripole emission contribution. As mentioned earlier, it vanishes in all collinear limits for timelike splittings. However, for spacelike splitting kinematics it is quite nontrivial. Eq. (4.15) contains a sum over two types of tripoles, corresponding to the two different kinematic configurations where $\lambda_{i k}=1$ and $\lambda_{i k}=0$. In the first situation, $\{i, k, q\}$ are outgoing (figure 2e); thus, according to table 1 , we consider the analytic continuation of the tripole term to the $A_{5}$ region. Since we set $j=1$, the tripole contributions in the collinear limit come from the $i \leftrightarrow j$ terms in eq. (3.16), and from the region where $z \equiv z_{k}^{i 1} \rightarrow 1$. Because the $A_{5}$ region is equivalent to the $A_{0}$ region, we find

$$
\begin{aligned}
\lim _{z, \bar{z} \rightarrow 1}\left[\left.\boldsymbol{S}_{a,\{i, 1, k\}}^{+,(2)}\right|_{A_{5}}\right]= & \boldsymbol{T}_{1}^{a_{1}} \frac{1}{\sqrt{-x_{q}}\langle 1 q\rangle}\left(\frac{\mu^{2}}{x_{q} s_{1 q}}\right)^{2 \epsilon} \exp [2 \mathrm{i} \pi \epsilon] 2 \boldsymbol{T}_{i}^{a_{i}} \boldsymbol{T}_{k}^{a_{k}} \\
& \times \lim _{z, \bar{z} \rightarrow 1}\left[f^{a a_{i} b} f^{b a_{1} a_{k}} D_{1}(1-z, 1-\bar{z})+f^{a a_{1} b} f^{b a_{k} a_{i}} D_{2}(1-z, 1-\bar{z})\right],
\end{aligned}
$$

with (see eq. (4.10))

$$
\lim _{z, \bar{z} \rightarrow 1} D_{1}(1-z, 1-\bar{z})=0, \quad \lim _{z, \bar{z} \rightarrow 1} D_{2}(1-z, 1-\bar{z})=0 .
$$

We conclude that for $\lambda_{i k}=1, \boldsymbol{S}_{a,\{i, 1, k\}}^{+,(2)}$ vanishes in the collinear limit.

For the second type of tripole with $\lambda_{i k}=0$, either $p_{i}$ or $p_{k}$ is incoming, cf. figure $2 \mathrm{~d}$. Without loss of generality, we label the incoming leg by $k$ and consider the analytic continuation of the tripole term to the $A_{1}$ region where $\{1, k\}$ are incoming and $\{i, q\}$ are outgoing. In this region, the collinear limit of $\boldsymbol{S}_{a,\{i, 1, k\}}^{+,(2)}$ is obtained from the collinear limit 
of its $A_{1}$ discontinuity.

$$
\begin{aligned}
\lim _{z, \bar{z} \rightarrow 1}\left[\left.\boldsymbol{S}_{a,\{i, 1, k\}}^{+,(2)}\right|_{A_{1}}\right]= & \lim _{z, \bar{z} \rightarrow 1} \operatorname{disc}_{A_{1}} \boldsymbol{S}_{a,\{i, 1, k\}}^{+,(2)} \\
= & \boldsymbol{T}_{1}^{a_{1}} \frac{1}{\sqrt{-x_{q}}\langle 1 q\rangle}\left(\frac{\mu^{2}}{x_{q} s_{1 q}}\right)^{2 \epsilon} \exp [-2 \mathrm{i} \pi \epsilon] \\
& \times 2 \boldsymbol{T}_{i}^{a_{i}} \boldsymbol{T}_{k}^{a_{k}} \lim _{z, \bar{z} \rightarrow 1}\left[f^{a a_{i} b} f^{b a_{1} a_{k}} \operatorname{disc}_{A_{1}} D_{1}(1-z, 1-\bar{z})\right. \\
& \left.\quad+f^{a a_{1} b} f^{b a_{k} a_{i}} \operatorname{disc}_{A_{1}} D_{2}(1-z, 1-\bar{z})\right],
\end{aligned}
$$

where

$$
\begin{aligned}
\lim _{z, \bar{z} \rightarrow 1} \operatorname{disc}_{A_{1}} D_{1}(1-z, 1-\bar{z})= & 4 \pi^{2}\left(\frac{1}{\epsilon^{2}}+\frac{2 \mathrm{i} \pi}{\epsilon}-14 \zeta_{2}\right) \\
\lim _{z, \bar{z} \rightarrow 1} \operatorname{disc}_{A_{1}} D_{2}(1-z, 1-\bar{z})= & 2 \mathrm{i} \pi\left\{\frac{\log |1-z|^{2}}{\epsilon^{2}}+2 \mathrm{i} \pi \frac{\log |1-z|^{2}}{\epsilon}\right. \\
& \left.+\frac{1}{3} \log \left(\frac{1-z}{1-\bar{z}}\right)\left[\log ^{2}\left(\frac{1-z}{1-\bar{z}}\right)+4 \pi^{2}\right]+4 \zeta_{3}-14 \zeta_{2} \log |1-z|^{2}\right\}
\end{aligned}
$$

and

$$
\log (1-z)=\log \left(1-z_{k}^{i 1}\right)=\log z_{k}^{1 i}, \quad \log (1-\bar{z})=\log \bar{z}_{k}^{1 i} .
$$

Applying the color Jacobi identity, we decompose the color factors in eq. (4.21) into structures that are even and odd under the $i \leftrightarrow k$ interchange,

$$
\begin{aligned}
f^{a a_{i} b} f^{b a_{1} a_{k}} & =-\frac{1}{2}\left(T_{q}^{a_{i}} T_{q}^{a_{k}}+T_{q}^{a_{k}} T_{q}^{a_{i}}\right)_{a a_{1}}+\frac{1}{2} f^{b a_{k} a_{i}}\left(T_{q}^{b}\right)_{a a_{1}}, \\
f^{a a_{1} b} f^{b a_{k} a_{i}} & =-f^{b a_{k} a_{i}}\left(T_{q}^{b}\right)_{a a_{1}} .
\end{aligned}
$$

Summing over all external legs, we obtain the two-loop tripole contribution to the splitting amplitude, written in terms of $1-z_{k}^{i 1}=z_{k}^{1 i}$,

$$
\begin{aligned}
&\left.\mathbf{S p}^{(2)}\right|_{\text {tripole }} \stackrel{q-\text { soft }}{\simeq}-\left.\frac{1}{4} \sum_{\text {tripoles }} \boldsymbol{S}_{a,\{i, 1, k\}}^{+,(2)}\right|_{q \| p_{1}} \\
&=\left(\frac{\mu^{2}}{x_{q} s_{1 q}}\right)^{2 \epsilon} \sum_{i \neq k \neq 1} \delta_{0, \lambda_{i k}} \delta_{1, \lambda_{1 k}}\left\{f^{b a_{k} a_{i}} \boldsymbol{T}_{q}^{b} \boldsymbol{T}_{k}^{a_{k}} \boldsymbol{T}_{i}^{a_{i}}\right. \\
& \times\left[\frac{1}{\epsilon^{2}}\left(\mathrm{i} \pi \log v_{k}^{1 i}-\pi^{2}\right)-\frac{\mathrm{i} \pi^{3}}{3} \log v_{k}^{1 i}+4 \mathrm{i} \pi \zeta_{3}+30 \zeta_{4}+\frac{8 \pi}{3}\left(\arg \left(z_{k}^{1 i}\right)^{3}-\pi^{2} \arg \left(z_{k}^{1 i}\right)\right)\right] \\
&\left.+\left[\left(\boldsymbol{T}_{q} \cdot \boldsymbol{T}_{i}\right)\left(\boldsymbol{T}_{q} \cdot \boldsymbol{T}_{k}\right)+\left(\boldsymbol{T}_{q} \cdot \boldsymbol{T}_{k}\right)\left(\boldsymbol{T}_{q} \cdot \boldsymbol{T}_{i}\right)\right]\left(\frac{\pi^{2}}{\epsilon^{2}}-30 \zeta_{4}\right)\right\} \mathbf{S p}^{(0)},
\end{aligned}
$$

where $\arg (z) \equiv \frac{1}{2 \mathrm{i}} \log \frac{z}{\bar{z}} \in(-\pi, \pi]$ is the argument of $z$, and the $\delta_{1, \lambda_{1 k}}$ is present to enforce our choice that we always label the incoming legs by $\{1, k\}$. Furthermore, we have expanded 
the phase factor $\exp [-2 \mathrm{i} \pi \epsilon]$ in $\epsilon$ and only keep overall terms to order $\mathcal{O}\left(\epsilon^{0}\right)$. As before, the kinematic dependence is given in terms of

$$
v_{k}^{1 i}=\frac{s_{i k} s_{1 q}}{s_{1 i} s_{k q}}, \quad z_{k}^{1 i}=\frac{\langle k i\rangle\langle 1 q\rangle}{\langle 1 i\rangle\langle k q\rangle} .
$$

The soft-collinear tripole term $\left.\mathbf{S} \mathbf{p}^{(2)}\right|_{\text {tripole }}$ in eq. (4.27) depends on the color and kinematics of the non-collinear tripole partons. It therefore explicitly breaks strict collinear factorization of scattering amplitudes. The factorization-breaking $1 / \epsilon$ poles in the two-loop splitting amplitude were given previously [33]. In the soft limit, they agree with the $1 / \epsilon$ poles in eq. (4.27). The two-loop finite terms in eq. (4.27) constitute a new result.

Our results also have implications for factorization violation at the level of the cross section. More explicitly, let us consider the perturbative expansion of the squared splitting probability, $|\mathbf{S p}|^{2}$. At leading order, $\mathcal{O}\left(\alpha_{s}\right)$ and at next-to-leading order, $\mathcal{O}\left(\alpha_{s}^{2}\right)$, strict factorization holds. At next-to-leading order, as mentioned earlier, it holds because the $\lambda_{k q}$ dependence is purely imaginary and cancels from the interference between tree and oneloop, $\mathbf{S} \mathbf{p}^{(0)} \mathbf{S} \mathbf{p}^{(1) \dagger}+\mathbf{S} \mathbf{p}^{(0) \dagger} \mathbf{S} \mathbf{p}^{(1)}$. Now let us examine the situation at $\mathcal{O}\left(\alpha_{s}^{3}\right)$. Factorizationbreaking contributions come from both $\left|\mathbf{S} \mathbf{p}^{(1)}\right|^{2}$ and the interference between $\mathbf{S p}^{(2)}$ and $\mathrm{Sp}^{(0)}$.

We first examine the interference of two one-loop splitting amplitudes in the soft limit, $\left|\mathbf{S} \mathbf{p}^{(1)}\right|^{2}$. Employing eq. (4.18)), we see that it does not fully factorize, and the nonfactorizing part contains a double pole in $\epsilon$,

$$
\begin{aligned}
\left|\mathbf{S p}^{(1)}\right|_{\text {non-fac. }}^{2} \stackrel{q-\text { soft }}{\simeq} \sum_{i \neq k \neq 1} \delta_{0, \lambda_{i k}} \mathbf{S p}^{(0) \dagger}\{ & {\left[-\left(\boldsymbol{T}_{q} \cdot \boldsymbol{T}_{i}\right)\left(\boldsymbol{T}_{q} \cdot \boldsymbol{T}_{k}\right)-\left(\boldsymbol{T}_{q} \cdot \boldsymbol{T}_{k}\right)\left(\boldsymbol{T}_{q} \cdot \boldsymbol{T}_{i}\right)\right] } \\
& \left.\times\left(\frac{\mu^{2}}{x_{q} s_{1 q}}\right)^{2 \epsilon}\left[\frac{\pi^{2}}{\epsilon^{2}}-15 \zeta_{4}+\mathcal{O}(\epsilon)\right]\right\} \mathbf{S p}^{(0)} .
\end{aligned}
$$

Now we consider the interference between $\mathbf{S} \mathbf{p}^{(2)}$ and $\mathbf{S} \mathbf{p}^{(0)}$. The contributions of the two-loop dipole terms in $\mathbf{S} \mathbf{p}^{(2)}$ factorize at the level of the cross section, for the same reason that strict factorization holds at $\mathcal{O}\left(\alpha_{s}^{2}\right)$. The two-loop tripole term in eq. (4.27) contains both Hermitian and anti-Hermitian parts. The latter cancel out in the squared splitting amplitude. In the final answer for $|\mathbf{S p}|^{2}$, the factorization-breaking $1 / \epsilon^{2}$ pole in $\left|\mathbf{S p}^{(1)}\right|^{2}$ is cancelled by the one in $\left\{\left.\mathbf{S p}^{(0) \dagger} \mathbf{S} \mathbf{p}^{(2)}\right|_{\text {tripole }}+\right.$ h.c. $\}$. Combining all remaining terms, we find,

$$
\begin{aligned}
& \left.\mathbf{S p}^{\dagger} \mathbf{S p}\right|_{\text {non-fac. }} ^{\underset{q-\text { soft }}{\simeq}} \bar{a}^{2} g_{s}^{2} \sum_{i \neq k \neq 1} \delta_{0, \lambda_{i k}} \mathbf{S p}^{(0) \dagger}\left\{\left[\left(\boldsymbol{T}_{q} \cdot \boldsymbol{T}_{i}\right)\left(\boldsymbol{T}_{q} \cdot \boldsymbol{T}_{k}\right)+\left(\boldsymbol{T}_{q} \cdot \boldsymbol{T}_{k}\right)\left(\boldsymbol{T}_{q} \cdot \boldsymbol{T}_{i}\right)\right]\left(-15 \zeta_{4}\right)\right. \\
& \left.+2 \pi \mathrm{i} \delta_{1, \lambda_{1 k}} f^{b a_{k} a_{i}} \boldsymbol{T}_{q}^{b} \boldsymbol{T}_{k}^{a_{k}} \boldsymbol{T}_{i}^{a_{i}}\left(\frac{\mu^{2}}{x_{q} s_{1 q}}\right)^{2 \epsilon}\left[\left(\frac{1}{\epsilon^{2}}-2 \zeta_{2}\right) \log v_{k}^{1 i}+4 \zeta_{3}\right]\right\} \mathbf{S p}^{(0)}+\mathcal{O}\left(\bar{a}^{4}\right) .
\end{aligned}
$$

Notice that the first line in eq. (4.30) does not contain the factor of $\delta_{1, \lambda_{1 k}}$ present in eq. (4.27); removing it absorbs a factor of two from the '+h.c.' terms. Also recall that in our conventions the structure constants $f^{a b c}$ are purely imaginary, so in the second line in 
eq. (4.30) the '+h.c.' terms result in a projection onto the kinematic terms containing an explicit 'i'.

The color structure in the second line in eq. (4.30) can be rewritten as a commutator, $\left[\left(\boldsymbol{T}_{q} \cdot \boldsymbol{T}_{i}\right),\left(\boldsymbol{T}_{q} \cdot \boldsymbol{T}_{k}\right)\right]$. When eq. (4.30) is sandwiched between tree amplitudes, $\left\langle\mathcal{M}_{n}^{(0)}|\cdots| \mathcal{M}_{n}^{(0)}\right\rangle$, and a color sum is performed, the Hermiticity of the operators $\boldsymbol{T}_{q} \cdot \boldsymbol{T}_{i}$ allows one to conclude that the color sum vanishes [34]. (A similar cancellation occurs for the $1 / \epsilon$ pole with the same color structure, which appears in two-loop four-point amplitudes [73] but cancels in the color-summed cross section [74].)

We conclude that for pure QCD splitting processes at order $g \times g^{5}$, or $\mathcal{O}\left(\alpha_{s}^{3}\right)$, potential factorization violation comes from the finite term in the first line in eq. (4.30), which has not been discussed before. We speculate that at next-to-next-to-next-to-leading order (NNNLO) in QCD, integrating over the phase space of the collinear splitting can give rise to soft-collinear poles which depend on the color charge of non-collinear partons entering the process. Such poles cannot be canceled by the conventional counterterms associated with renormalization of the parton distribution functions (PDFs), which by definition are process independent. (The failure of strict factorization at NNNLO for non-inclusive hadron collider processes has been argued previously, based partly on the structure of $1 / \epsilon$ pole terms associated with Coulomb gluon exchanges [34-36].) An interesting example that can contain such factorization-violating contributions is the NNNLO QCD corrections to dijet production at hadron colliders. While the full NNNLO QCD corrections might still be far away, a shortcut to revealing the factorization-breaking terms is through the study of precision hadron collider event shapes [75], where NNNLO corrections including logarithms in the event-shape variable are within reach. We leave the investigation of these important issues to future work.

\section{Conclusions}

In this paper we computed the exact kinematic and color dependence of soft-gluon emission in massless gauge theory at the two loop level. While the dipole terms have a simple kinematic dependence and had been computed previously [25, 26], the subleading-color tripole terms are new, and they depend in an intricate way on a rescaling-invariant cross ratio.

Using the soft-collinear limit of our results, we could study the soft limits of two-loop collinear splitting amplitudes for both timelike and spacelike kinematics. The timelike behavior was understood previously [26, 47, 71, 72]. In the spacelike case, the infrared singular parts of the two-loop splitting amplitudes were obtained before in ref. [33], with which we find full agreement. Our new results for this case are the finite contributions, provided in eq. (4.27). Note that eq. (4.27) is non-zero only when the non-collinear tripole partons $i$ and $k$ are spacelike separated. Thus, including the collinear parton 1, there must be two partons in the initial state to get a contribution (i.e. deep inelastic scattering does not qualify, while hadronic collisions do). Both eqs. (4.18) and (4.27) violate strict collinear factorization [33,34], in the sense that the splitting amplitudes depend on the color and/or kinematics of some non-collinear hard partons in the process. For dipole emission, eq. (4.18), factorization violation only exists in the imaginary part. The real part 
preserves strict collinear factorization, after using color conservation. Also, the violation in the dipole emission contribution is independent of kinematics (as a multiple of tree-level splitting). On the other hand, the tripole emission, eq. (4.27), violates strict collinear factorization with both color and kinematic correlations at the amplitude level.

The violation of strict collinear factorization is related to issues in the breakdown of factorization for transverse-momentum-dependent PDFs [76-79], double parton scattering [80-82], and the resummation of logarithms in non-global observables [35, 36, 83, 84] or event shapes at hadron colliders [75, 85-87]. It has been studied in an effective field theory framework which isolates the relevant physical degree of freedom, the so-called "Glauber", or "Coulomb" mode, which is responsible for the breaking of collinear factorization [88-90]. Our explicit result for the two-loop soft factor manifestly shows the breakdown of collinear factorization at the amplitude level in the soft limit. Furthermore, we have demonstrated that potential factorization-breaking terms at the cross-section level also appear as $\mathcal{O}\left(\alpha_{s}^{3}\right)$ contributions to splitting in pure QCD processes such as dijet production at hadron colliders. It would be interesting to understand the relation between the factorization-breaking terms in the soft factor and the Glauber gluon, by extending the one-loop soft current analysis in ref. [88] to two loops.

At large $N_{c}$, the two-loop soft-gluon emission factor has already been applied to compute the threshold soft corrections to the production of a color neutral state at hadron colliders at NNNLO [91, 92]. The results of this paper can be applied as a building block in precision calculations of generic jet cross sections, including full color dependence.

The analytic behavior of the tripole emission terms for soft-gluon emission at two loops is quite intricate. The functions $D_{1}$ and $D_{2}$ must be well-defined and unambiguous, not only in the Euclidean region $A_{0}$, but also in other regions obtained by analytic continuation, such as $A_{1}$. While any SVHPL is unambiguous in the Euclidean region, it may develop branch cuts in Minkowski regions. The requirement that these cuts cancel when this region is kinematically accessible, as is the case here, is a strong constraint which may be useful for constraining higher-loop results by their analytic properties (bootstrapping). We remark that the quadrupole terms that couple four hard legs in the soft anomalous dimension matrix $[43,59]$ have to obey this same constraint. Both cases involve four massless momentum vectors in a scattering process with (generically) more particles. (In our case, one of the vectors is soft, but that does not matter for the definition of $z$ and $\bar{z}$ since they are rescaling invariant.) In both cases the Minkowski kinematics can be smoothly continued around the real axis for $z>1$, and the physical result must be continuous during this process, implying a branch cut cancellation analogous to eq. (3.44), for example. On the other hand, the soft anomalous dimension quadrupole terms have a $z \leftrightarrow \bar{z}$ parity symmetry. (Parity is not a symmetry of the soft factor for emitting a positive-helicity gluon, because it maps it to negative-helicity emission.) After imposing parity in the Euclidean region, branch cut cancellation in Minkowski regions is likely to be automatic.

Soft emission at higher loops should reveal additional structures. For example, the three-loop soft emission factor will include a purely non-Abelian quadrupole term (see figure 3(a)), whose finite part we expect to have a uniform transcendental weight 6 , and nontrivial kinematic dependence on several cross ratios. It will also contain a tripole term 


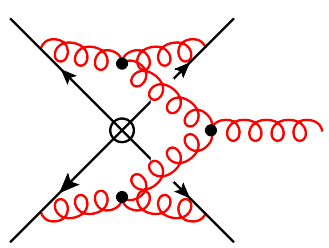

(a)

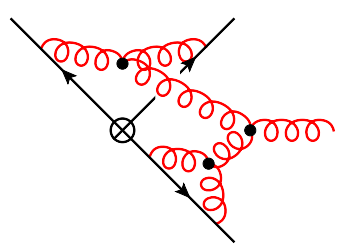

(b)

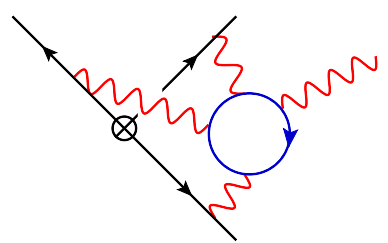

(c)

Figure 3. Representative diagrams for the (a) three-loop quadrupole, (b) three-loop tripole, and (c) three-loop Abelian soft factors.

(figure 3(b)) which will depend on the matter content, like the dipole term at two loops, but with kinematic dependence on $z_{k}^{i j}$ similar to the two-loop tripole term. We expect this term to have non-uniform weight, except in the case of $\mathcal{N}=4$ SYM. Of course, the threeloop dipole term must have the same simple kinematic dependence as the one and two loop dipole terms. Finally, in massless QED, the soft emission factor for unrenormalized amplitudes should get its first quantum correction at three loops, from a light-by-light scattering contribution shown in figure 3(c). This Abelian contribution can have both tripole and dipole terms. These terms cannot be associated with a tree-level soft emission factor because they have a different dependence on the charge of the hard fermion, versus the charge of the massless fermion in the loop. Similar contributions in nonabelian gauge theory will involve the quartic Casimir operator.

In summary, we have presented the soft factor required for single soft-gluon emission in two-loop gauge theory amplitudes. Applications of our results include construction of infrared subtraction terms for NNNLO jet cross sections (see also ref. [93] for the tree-level triple soft emission contribution, and ref. [94] for the tree-level quadruple collinear splitting contribution), the calculation of QCD corrections at NNNLO in the soft-gluon approximation beyond leading-color, the soft approximation of two-loop gauge theory amplitudes, and the quantitative study of factorization violation.

\section{Acknowledgments}

We thank Yu Jiao Zhu for checking part of the results in this paper. We also thank Falko Dulat for analytic continuation assistance and Claude Duhr, Andrew McLeod, and Giulia Zanderighi for useful discussions. This work is supported by the Department of Energy under contract DE-AC02-76SF00515. HXZ is supported by the National Natural Science Foundation of China under contract No. 11975200. This research received funding from the European Research Council (ERC) under the European Union's Horizon 2020 research and innovation programme (grant agreement No. 725110), "Novel structures in scattering amplitudes".

Open Access. This article is distributed under the terms of the Creative Commons Attribution License (CC-BY 4.0), which permits any use, distribution and reproduction in any medium, provided the original author(s) and source are credited. 


\section{References}

[1] J.M. Drummond, J. Henn, V.A. Smirnov and E. Sokatchev, Magic identities for conformal four-point integrals, JHEP 01 (2007) 064 [hep-th/0607160] [INSPIRE].

[2] L.F. Alday and J.M. Maldacena, Gluon scattering amplitudes at strong coupling, JHEP 06 (2007) 064 [arXiv:0705.0303] [INSPIRE].

[3] J.M. Drummond, J. Henn, G.P. Korchemsky and E. Sokatchev, Dual superconformal symmetry of scattering amplitudes in $N=4$ super-Yang-Mills theory, Nucl. Phys. B 828 (2010) 317 [arXiv:0807.1095] [INSPIRE].

[4] M.L. Mangano and S.J. Parke, Multiparton amplitudes in gauge theories, Phys. Rept. 200 (1991) 301 [hep-th/0509223] [INSPIRE].

[5] Z. Bern, L.J. Dixon, D.C. Dunbar and D.A. Kosower, One loop n point gauge theory amplitudes, unitarity and collinear limits, Nucl. Phys. B 425 (1994) 217 [hep-ph/9403226] [INSPIRE].

[6] L.J. Dixon, Calculating scattering amplitudes efficiently, in QCD and beyond. Proceedings, Theoretical Advanced Study Institute in Elementary Particle Physics, TASI-95, Boulder, CO, USA, 4-30 June 1995, pg. 539 [hep-ph/9601359] [INSPIRE].

[7] A. Bassetto, M. Ciafaloni and G. Marchesini, Jet structure and infrared sensitive quantities in perturbative QCD, Phys. Rept. 100 (1983) 201 [INSPIRE].

[8] F.A. Berends and W.T. Giele, Multiple soft gluon radiation in parton processes, Nucl. Phys. B 313 (1989) 595 [INSPIRE].

[9] Z. Bern, V. Del Duca, W.B. Kilgore and C.R. Schmidt, The infrared behavior of one loop QCD amplitudes at next-to-next-to leading order, Phys. Rev. D 60 (1999) 116001 [hep-ph/9903516] [INSPIRE].

[10] W.T. Giele and E.W.N. Glover, Higher order corrections to jet cross-sections in $e^{+} e^{-}$ annihilation, Phys. Rev. D 46 (1992) 1980 [INSPIRE].

[11] W.T. Giele, E.W.N. Glover and D.A. Kosower, Higher order corrections to jet cross-sections in hadron colliders, Nucl. Phys. B 403 (1993) 633 [hep-ph/9302225] [INSPIRE].

[12] J.M. Campbell and E.W.N. Glover, Double unresolved approximations to multiparton scattering amplitudes, Nucl. Phys. B 527 (1998) 264 [hep-ph/9710255] [INSPIRE].

[13] S. Catani and M. Grazzini, Infrared factorization of tree level QCD amplitudes at the next-to-next-to-leading order and beyond, Nucl. Phys. B 570 (2000) 287 [hep-ph/9908523] [INSPIRE].

[14] J.C. Collins, D.E. Soper and G.F. Sterman, Factorization of hard processes in QCD, Adv. Ser. Direct. High Energy Phys. 5 (1989) 1 [hep-ph/0409313] [INSPIRE].

[15] G.F. Sterman, Partons, factorization and resummation, TASI 95, in QCD and beyond. Proceedings, Theoretical Advanced Study Institute in Elementary Particle Physics, TASI-95, Boulder, CO, U.S.A., 4-30 June 1995, pg. 327 [hep-ph/9606312] [INSPIRE].

[16] G.F. Sterman, QCD and jets, in Physics in $D \geq 4$. Proceedings, Theoretical Advanced Study Institute in elementary particle physics, TASI 2004, Boulder, CO, U.S.A., 6 June-2 July 2004, pg. 67 [hep-ph/0412013] [INSPIRE]. 
[17] Z. Bern, G. Chalmers, L.J. Dixon and D.A. Kosower, One loop $N$ gluon amplitudes with maximal helicity violation via collinear limits, Phys. Rev. Lett. 72 (1994) 2134 [hep-ph/9312333] [INSPIRE].

[18] Z. Bern, L.J. Dixon and D.A. Kosower, One loop amplitudes for $e^{+} e^{-}$to four partons, Nucl. Phys. B 513 (1998) 3 [hep-ph/9708239] [INSPIRE].

[19] L.J. Dixon, J.M. Drummond, M. von Hippel and J. Pennington, Hexagon functions and the three-loop remainder function, JHEP 12 (2013) 049 [arXiv:1308.2276] [INSPIRE].

[20] S. Caron-Huot, L.J. Dixon, F. Dulat, M. von Hippel, A.J. McLeod and G. Papathanasiou, Six-gluon amplitudes in planar $N=4$ super-Yang-Mills theory at six and seven loops, JHEP 08 (2019) 016 [arXiv: 1903.10890] [INSPIRE].

[21] S. Weinberg, Infrared photons and gravitons, Phys. Rev. 140 (1965) B516 [InSPIRE].

[22] Z. Bern and G. Chalmers, Factorization in one loop gauge theory, Nucl. Phys. B 447 (1995) 465 [hep-ph/9503236] [INSPIRE].

[23] Z. Bern, V. Del Duca and C.R. Schmidt, The infrared behavior of one loop gluon amplitudes at next-to-next-to-leading order, Phys. Lett. B 445 (1998) 168 [hep-ph/9810409] [INSPIRE].

[24] S. Catani and M. Grazzini, The soft gluon current at one loop order, Nucl. Phys. B 591 (2000) 435 [hep-ph/0007142] [INSPIRE].

[25] Y. Li and H.X. Zhu, Single soft gluon emission at two loops, JHEP 11 (2013) 080 [arXiv: 1309.4391] [INSPIRE].

[26] C. Duhr and T. Gehrmann, The two-loop soft current in dimensional regularization, Phys. Lett. B 727 (2013) 452 [arXiv: 1309.4393] [INSPIRE].

[27] L.J. Dixon, J.M. Drummond and J.M. Henn, Bootstrapping the three-loop hexagon, JHEP 11 (2011) 023 [arXiv: 1108.4461] [INSPIRE].

[28] Z. Bern, L.J. Dixon and V.A. Smirnov, Iteration of planar amplitudes in maximally supersymmetric Yang-Mills theory at three loops and beyond, Phys. Rev. D 72 (2005) 085001 [hep-th/0505205] [INSPIRE].

[29] N. Arkani-Hamed, J.L. Bourjaily, F. Cachazo, A.B. Goncharov, A. Postnikov and J. Trnka, Grassmannian geometry of scattering amplitudes, Cambridge University Press, Cambridge, U.K. (2016) [arXiv:1212.5605] [INSPIRE].

[30] A.V. Kotikov and L.N. Lipatov, On the highest transcendentality in $N=4$ SUSY, Nucl. Phys. B 769 (2007) 217 [hep-th/0611204] [INSPIRE].

[31] S. Abreu, L.J. Dixon, E. Herrmann, B. Page and M. Zeng, The two-loop five-point amplitude in $N=4$ super-Yang-Mills theory, Phys. Rev. Lett. 122 (2019) 121603 [arXiv:1812.08941] [INSPIRE].

[32] D. Chicherin, T. Gehrmann, J.M. Henn, P. Wasser, Y. Zhang and S. Zoia, Analytic result for a two-loop five-particle amplitude, Phys. Rev. Lett. 122 (2019) 121602 [arXiv:1812.11057] [INSPIRE].

[33] S. Catani, D. de Florian and G. Rodrigo, Space-like (versus time-like) collinear limits in QCD: is factorization violated?, JHEP 07 (2012) 026 [arXiv: 1112.4405] [INSPIRE].

[34] J.R. Forshaw, M.H. Seymour and A. Siodmok, On the breaking of collinear factorization in QCD, JHEP 11 (2012) 066 [arXiv:1206.6363] [INSPIRE]. 
[35] J.R. Forshaw, A. Kyrieleis and M.H. Seymour, Super-leading logarithms in non-global observables in QCD, JHEP 08 (2006) 059 [hep-ph/0604094] [INSPIRE].

[36] J.R. Forshaw, A. Kyrieleis and M.H. Seymour, Super-leading logarithms in non-global observables in QCD: colour basis independent calculation, JHEP 09 (2008) 128 [arXiv: 0808.1269] [INSPIRE].

[37] S. Catani and M.H. Seymour, A general algorithm for calculating jet cross-sections in NLO QCD, Nucl. Phys. B 485 (1997) 291 [Erratum ibid. B 510 (1998) 503] [hep-ph/9605323] [INSPIRE].

[38] N. Kidonakis, G. Oderda and G.F. Sterman, Evolution of color exchange in QCD hard scattering, Nucl. Phys. B 531 (1998) 365 [hep-ph/9803241] [INSPIRE].

[39] S.M. Aybat, L.J. Dixon and G.F. Sterman, The two-loop soft anomalous dimension matrix and resummation at next-to-next-to leading pole, Phys. Rev. D 74 (2006) 074004 [hep-ph/0607309] [INSPIRE].

[40] L.J. Dixon, L. Magnea and G.F. Sterman, Universal structure of subleading infrared poles in gauge theory amplitudes, JHEP 08 (2008) 022 [arXiv:0805.3515] [INSPIRE].

[41] E. Gardi and L. Magnea, Factorization constraints for soft anomalous dimensions in QCD scattering amplitudes, JHEP 03 (2009) 079 [arXiv:0901.1091] [INSPIRE].

[42] T. Becher and M. Neubert, Infrared singularities of scattering amplitudes in perturbative QCD, Phys. Rev. Lett. 102 (2009) 162001 [Erratum ibid. 111 (2013) 199905] [arXiv:0901.0722] [INSPIRE].

[43] Ø. Almelid, C. Duhr and E. Gardi, Three-loop corrections to the soft anomalous dimension in multileg scattering, Phys. Rev. Lett. 117 (2016) 172002 [arXiv:1507.00047] [INSPIRE].

[44] A.V. Manohar, T. Mehen, D. Pirjol and I.W. Stewart, Reparameterization invariance for collinear operators, Phys. Lett. B 539 (2002) 59 [hep-ph/0204229] [INSPIRE].

[45] I. Feige and M.D. Schwartz, Hard-soft-collinear factorization to all orders, Phys. Rev. D 90 (2014) 105020 [arXiv: 1403.6472] [INSPIRE].

[46] A.J. Larkoski, D. Neill and I.W. Stewart, Soft theorems from effective field theory, JHEP 06 (2015) 077 [arXiv:1412.3108] [INSPIRE].

[47] S.D. Badger and E.W.N. Glover, Two loop splitting functions in QCD, JHEP 07 (2004) 040 [hep-ph/0405236] [INSPIRE].

[48] P. Nogueira, Automatic Feynman graph generation, J. Comput. Phys. 105 (1993) 279.

[49] R.N. Lee, Presenting LiteRed: a tool for the Loop InTEgrals REDuction, arXiv:1212.2685 [INSPIRE].

[50] K.G. Chetyrkin and F.V. Tkachov, Integration by parts: the algorithm to calculate $\beta$-functions in 4 loops, Nucl. Phys. B 192 (1981) 159 [INSPIRE].

[51] Z. Bern, L.J. Dixon and D.A. Kosower, Dimensionally regulated pentagon integrals, Nucl. Phys. B 412 (1994) 751 [hep-ph/9306240] [INSPIRE].

[52] T. Gehrmann and E. Remiddi, Differential equations for two loop four point functions, Nucl. Phys. B 580 (2000) 485 [hep-ph/9912329] [INSPIRE].

[53] J.M. Henn, Multiloop integrals in dimensional regularization made simple, Phys. Rev. Lett. 110 (2013) 251601 [arXiv:1304.1806] [INSPIRE]. 
[54] G. 't Hooft and M.J.G. Veltman, Regularization and renormalization of gauge fields, Nucl. Phys. B 44 (1972) 189 [INSPIRE].

[55] Z. Bern and D.A. Kosower, The computation of loop amplitudes in gauge theories, Nucl. Phys. B 379 (1992) 451 [INSPIRE].

[56] Z. Bern, A. De Freitas, L.J. Dixon and H.L. Wong, Supersymmetric regularization, two loop QCD amplitudes and coupling shifts, Phys. Rev. D 66 (2002) 085002 [hep-ph/0202271] [INSPIRE].

[57] C. Anastasiou, Z. Bern, L.J. Dixon and D.A. Kosower, Planar amplitudes in maximally supersymmetric Yang-Mills theory, Phys. Rev. Lett. 91 (2003) 251602 [hep-th/0309040] [INSPIRE].

[58] V. Lysov, S. Pasterski and A. Strominger, Low's subleading soft theorem as a symmetry of QED, Phys. Rev. Lett. 113 (2014) 111601 [arXiv:1407.3814] [InSPIRE].

[59] Ø. Almelid, C. Duhr, E. Gardi, A. McLeod and C.D. White, Bootstrapping the QCD soft anomalous dimension, JHEP 09 (2017) 073 [arXiv: 1706.10162] [INSPIRE].

[60] A.B. Goncharov, M. Spradlin, C. Vergu and A. Volovich, Classical polylogarithms for amplitudes and Wilson loops, Phys. Rev. Lett. 105 (2010) 151605 [arXiv:1006.5703] [INSPIRE].

[61] D. Gaiotto, J. Maldacena, A. Sever and P. Vieira, Pulling the straps of polygons, JHEP 12 (2011) 011 [arXiv:1102.0062] [INSPIRE].

[62] F.C.S. Brown, Single-valued multiple polylogarithms in one variable, Compt. Rend. Acad. Sci. 338 (2004) 527.

[63] L.J. Dixon, C. Duhr and J. Pennington, Single-valued harmonic polylogarithms and the multi-Regge limit, JHEP 10 (2012) 074 [arXiv:1207.0186] [INSPIRE].

[64] E. Remiddi and J.A.M. Vermaseren, Harmonic polylogarithms, Int. J. Mod. Phys. A 15 (2000) 725 [hep-ph/9905237] [inSPIRE].

[65] C. Duhr and F. Dulat, PolyLogTools - polylogs for the masses, JHEP 08 (2019) 135 [arXiv: 1904.07279] [INSPIRE].

[66] Z. Bern, J.S. Rozowsky and B. Yan, Two loop four gluon amplitudes in $N=4$ super Yang-Mills, Phys. Lett. B 401 (1997) 273 [hep-ph/9702424] [INSPIRE].

[67] V.A. Smirnov, Analytical result for dimensionally regularized massive on-shell planar double box, Phys. Lett. B 524 (2002) 129 [hep-ph/0111160] [INSPIRE].

[68] J.B. Tausk, Nonplanar massless two loop Feynman diagrams with four on-shell legs, Phys. Lett. B 469 (1999) 225 [hep-ph/9909506] [INSPIRE].

[69] S. Catani, The singular behavior of QCD amplitudes at two loop order, Phys. Lett. B 427 (1998) 161 [hep-ph/9802439] [INSPIRE].

[70] G.F. Sterman and M.E. Tejeda-Yeomans, Multiloop amplitudes and resummation, Phys. Lett. B 552 (2003) 48 [hep-ph/0210130] [INSPIRE].

[71] Z. Bern, L.J. Dixon and D.A. Kosower, Two-loop $g \rightarrow$ gg splitting amplitudes in QCD, JHEP 08 (2004) 012 [hep-ph/0404293] [INSPIRE].

[72] C. Duhr, T. Gehrmann and M. Jaquier, Two-loop splitting amplitudes and the single-real contribution to inclusive Higgs production at $N^{3}$ LO, JHEP 02 (2015) 077 [arXiv:1411.3587] [INSPIRE]. 
[73] Z. Bern, A. De Freitas and L.J. Dixon, Two loop helicity amplitudes for gluon-gluon scattering in QCD and supersymmetric Yang-Mills theory, JHEP 03 (2002) 018 [hep-ph/0201161] [INSPIRE].

[74] E.W.N. Glover, C. Oleari and M.E. Tejeda-Yeomans, Two loop QCD corrections to gluon-gluon scattering, Nucl. Phys. B 605 (2001) 467 [hep-ph/0102201] [INSPIRE].

[75] A. Gao, H.T. Li, I. Moult and H.X. Zhu, Precision QCD event shapes at hadron colliders: the transverse energy-energy correlator in the back-to-back limit, Phys. Rev. Lett. 123 (2019) 062001 [arXiv: 1901.04497] [INSPIRE].

[76] C.J. Bomhof, P.J. Mulders and F. Pijlman, Gauge link structure in quark-quark correlators in hard processes, Phys. Lett. B 596 (2004) 277 [hep-ph/0406099] [INSPIRE].

[77] J. Collins and J.-W. Qiu, $k_{T}$ factorization is violated in production of high-transverse-momentum particles in hadron-hadron collisions, Phys. Rev. D 75 (2007) 114014 [arXiv: 0705.2141] [INSPIRE].

[78] W. Vogelsang and F. Yuan, Hadronic dijet imbalance and transverse-momentum dependent parton distributions, Phys. Rev. D 76 (2007) 094013 [arXiv:0708.4398] [INSPIRE].

[79] T.C. Rogers and P.J. Mulders, No generalized TMD-factorization in hadro-production of high transverse momentum hadrons, Phys. Rev. D 81 (2010) 094006 [arXiv:1001.2977] [INSPIRE].

[80] J.R. Gaunt and T. Kasemets, Transverse momentum dependence in double parton scattering, Adv. High Energy Phys. 2019 (2019) 3797394 [arXiv: 1812.09099] [INSPIRE].

[81] M. Diehl, J.R. Gaunt, P. Plößl and A. Schäfer, Two-loop splitting in double parton distributions, SciPost Phys. 7 (2019) 017 [arXiv: 1902.08019] [INSPIRE].

[82] M.G.A. Buffing, M. Diehl and T. Kasemets, Transverse momentum in double parton scattering: factorisation, evolution and matching, JHEP 01 (2018) 044 [arXiv:1708.03528] [INSPIRE].

[83] M. Balsiger, T. Becher and D.Y. Shao, Non-global logarithms in jet and isolation cone cross sections, JHEP 08 (2018) 104 [arXiv: 1803.07045] [INSPIRE].

[84] T. Becher, M. Neubert, L. Rothen and D.Y. Shao, Factorization and resummation for jet processes, JHEP 11 (2016) 019 [Erratum ibid. 05 (2017) 154] [arXiv:1605.02737] [INSPIRE].

[85] A. Banfi, G.P. Salam and G. Zanderighi, Resummed event shapes at hadron-hadron colliders, JHEP 08 (2004) 062 [hep-ph/0407287] [INSPIRE].

[86] I.W. Stewart, F.J. Tackmann and W.J. Waalewijn, The beam thrust cross section for Drell-Yan at NNLL order, Phys. Rev. Lett. 106 (2011) 032001 [arXiv:1005.4060] [INSPIRE].

[87] T. Becher and X. Garcia i Tormo, Factorization and resummation for transverse thrust, JHEP 06 (2015) 071 [arXiv: 1502.04136] [INSPIRE].

[88] I.Z. Rothstein and I.W. Stewart, An effective field theory for forward scattering and factorization violation, JHEP 08 (2016) 025 [arXiv: 1601.04695] [INSPIRE].

[89] M.D. Schwartz, K. Yan and H.X. Zhu, Collinear factorization violation and effective field theory, Phys. Rev. D 96 (2017) 056005 [arXiv:1703.08572] [InSPIRE].

[90] M.D. Schwartz, K. Yan and H.X. Zhu, Factorization violation and scale invariance, Phys. Rev. D 97 (2018) 096017 [arXiv:1801.01138] [InSPIRE]. 
[91] C. Anastasiou et al., Higgs boson gluon-fusion production at threshold in $N^{3} L O Q C D$, Phys. Lett. B 737 (2014) 325 [arXiv: 1403.4616] [INSPIRE].

[92] Y. Li, A. von Manteuffel, R.M. Schabinger and H.X. Zhu, Soft-virtual corrections to Higgs production at $N^{3} L O$, Phys. Rev. D 91 (2015) 036008 [arXiv:1412.2771] [INSPIRE].

[93] S. Catani, D. Colferai and A. Torrini, Triple (and quadruple) soft-gluon radiation in $Q C D$ hard scattering, JHEP 01 (2020) 118 [arXiv: 1908.01616] [INSPIRE].

[94] V. Del Duca, C. Duhr, R. Haindl, A. Lazopoulos and M. Michel, Tree-level splitting amplitudes for a quark into four collinear partons, JHEP 02 (2020) 189 [arXiv: 1912.06425] [INSPIRE]. 